\title{
Kauffman's clock lattice as a graph of perfect matchings: a formula for its height
}

\author{
Moshe Cohen* \\ Department of Electrical Engineering \\ Technion - Israel Institute of Technology \\ Haifa, Israel \\ mcohen@tx.technion.ac.il \\ Mina Teicher \\ Department of Mathematics and Computer Science \\ Bar-Ilan University \\ Ramat Gan, Israel \\ teicher@macs.biu.ac.il
}

Submitted: May 20, 2013; Accepted: Oct 29, 2014; Published: Nov 13, 2014

Mathematics Subject Classifications: 57M25, 57M15, 05C70, 05C90

\begin{abstract}
We give an algorithmic computation for the height of Kauffman's clock lattice obtained from a knot diagram with two adjacent regions starred and without crossing information specified. We show that this lattice is more familiarly the graph of perfect matchings of a bipartite graph obtained from the knot diagram by overlaying the two dual Tait graphs of the knot diagram. Furthermore we prove structural properties of the bipartite graph in general. This setting also makes evident applications to Chebyshev or harmonic knots, whose related bipartite graph is the popular grid graph, and to discrete Morse functions.
\end{abstract}

\section{Introduction}

There is a bijection between the set of all knot (and link) diagrams and the set of all signed plane graphs $G$. Spanning tree expansions of $G$ have been used to produce several models of use in knot theory: Kauffman [35] gives one for the Alexander polynomial; Thistlethwaite [54] for the Jones polynomial (related to work on the signed Tutte polynomial by Kauffman [36] and extended to work on the Bollobás-Riordan-Tutte polynomial

${ }^{*}$ Partially supported by the Oswald Veblen Fund and by the Minerva Foundation of Germany. 
by Dasbach, Futer, Kalfagianni, Lin, and Stoltzfus [21]); Greene [29] (of a different flavor) for the Heegaard Floer homology of the branched double cover of a knot; Ozsváth and Szabó [51] and Baldwin and Levine [6] (of this different flavor; analogous to different unpublished work by Ozsváth and Szabó) give some for knot Floer homology; and Wehrli [55], Champarnekar and Kofman [14] (independently), and Roberts [53] (of this different flavor; see also remarks by Jaeger [33]) for Khovanov homology.

There is a another bijection between the set of (rooted) spanning trees (or arborescences) of a plane graph $G$ and the set of perfect matchings (or dimer coverings) of a related plane bipartite graph $\Gamma$ that has been explored in previous work by the first author [16] and the first author with Dasbach and Russell [17], as well as in work by Kenyon, Propp, and Wilson [38], who say about this bijection:

This theorem, along with its proof, is a generalization of a result of Temperley (1974) which is discussed in problem 4.30 of [48, pp. 34, 104, 243-244]. The unweighted undirected generalization was independently discovered by Burton and Pemantle [12], who applied it to infinite graphs, and also by F. Y. $\mathrm{Wu}$, who included it in lecture notes for a course.

The related graph $\widehat{\Gamma}$ also appeared in work by Huggett, Moffatt, and Virdee [32]. The graph $\Gamma$ is currently being studied by Kravchenko and Polyak [44] for knots on a torus in relation to cluster algebras. Dimers themselves have been studied extensively, as well; see for example Kenyon's lecture notes [37] on the subject.

By the end of this present paper the authors hope that the reader will prefer the perfect matching model for $\Gamma$ below to the spanning tree model for $G$. In support of this we offer evidence that previous work in knot theory can be translated into concepts that are more regularly studied by graph theorists.

The primary example of this considered below is Kauffman's clock lattice $\mathcal{L}$ [35], which we translate into the graph $\mathcal{G}$ of perfect matchings of the plane bipartite graph $\Gamma$. This perspective offers beneficial insight to both sides: well-studied combinatorial tools can now be applied to knots, and some basic topological structure makes $\mathcal{G}$ easier to understand by directing its edges. In short this analogy allows for the height of the lattice to be seen as the diameter of $\mathcal{G}$, a topic of interest in work by Hernando, Hurtado, and Noy [30] and Athanasiadis and Roichman [3].

The work in this present paper is done for knot projections without crossing information; this corresponds to the unweighted graph $\Gamma$. One may obtain this crossing information by weighting the graph in one of several different ways, including Kauffman's "black and white holes" and the first author's previous work on this subject. It is currently unclear to to the authors whether there is one weighting that is more useful than all the others in every context.

Results. The main result of this paper, Theorem 56, states that this height can be computed combinatorially from the graph $\Gamma$ by counting the number of (square) faces within certain cycles $\left\{C_{i}\right\}$ that emerge in the discussion below. Specifically these cycles are constructed in Theorem 27 and arise from the unique minimum and unique maximum elements in the clock lattice $\mathcal{L}$ in Theorem 55 . 
Subection 8.1 gives an application of the main result to grid graphs, which appear often in graph theory literature. These are actually the balanced overlaid Tait graphs for harmonic knots.

Conjecturally, the authors of the present paper believe there is a relationship between the number of cycles of $\left\{C_{i}\right\}$ and the minimum number of local maxima in this particular knot diagram. Of course this provides an upper bound on the bridge number of the knot. This relationship is motivated by Abe's two main results in [1], discussed at the end of Section 3, and reaffirmed by new work on harmonic knots.

Lastly, Subsection 8.2 and specifically Proposition 70 establish the correspondence between perfect matchings of $\Gamma$ and discrete Morse functions on a 2-complex of the 2sphere whose 1-skeleton is the (unsigned) plane graph $G$ with a pair of specifically chosen critical cells.

Organization. The next Section 2 gives the construction for the balanced overlaid Tait graph $\Gamma$ and introduces the Periphery Proposition 3 which appears several times throughout the paper. The graph $\mathcal{G}$ of perfect matchings of $\Gamma$ is defined in Section 3. Some useful notions from graph theory are discussed in Section 4: connectivity and elementary graphs.

The main results begin in Section 5 with some operations that are used in the main construction Theorem 27. Further structural properties like leaf edges, accordions, and party hats are discussed in Section 6 (specifically Theorem 49), and some reduction moves are introduced to simplify $\Gamma$. The proof of the Main Theorem 56 in Section 7 is split into several lemmas based on these moves.

Finally several examples are discussed in Section 8, including a subsection on harmonic knots and a subsection on discrete Morse theory.

\section{The balanced overlaid Tait graph}

A knot $K$ is a circle $S^{1}$ embedded in $S^{3}=\mathbb{R}^{3} \cup\{\infty\}$. A link is the embedding of several copies of $S^{1}$. A knot or link diagram $D$ is the projection of the knot or link onto $\mathbb{R}^{2}$ with under- and over-crossing information. A theorem by Reidemeister in 1926 (see for example [47]) states that two diagrams represent the same knot if and only if there is a sequence of the three Reidemeister moves taking one diagram to the other.

The knot diagram considered without crossing information is a 4-regular plane graph called the projection graph (or the universe $U$ according to Kauffman [35]). By Euler's formula, there are two more faces than vertices; Kauffman chooses two adjacent faces to omit and marks these $*$ with stars. He then considers states: bijections between the set of vertices and the set of all un-starred faces. The state itself is depicted by placing markers at a corner of each crossing and in each face.

Ultimately, Kauffman uses the states on a universe to produce the Alexander polynomial $\Delta_{K}(t)$ of the knot $K$, which since its finding in 1923 has remained one of the most important classical knot invariants. It is precisely due to the Alexander module that the two starred faces must be adjacent. There are at least sixteen equivalent definitions of 
the Alexander polynomial, some of this redundancy owing perhaps to the different ways in which we can define Kauffman's states. One such way involving the construction below can be found in [17].

A state can be realized as a rooted spanning tree of a plane graph $G$ obtained from a diagram together with the complementary rooted spanning tree of the plane dual $G^{*}$ to this graph. One can obtain this signed Tait graph $G$ from a diagram by checkerboardcoloring its regions, taking the black regions to be the vertices, and taking signed edges corresponding to the crossings as in Figure 1.

$$
\text { positive }
$$
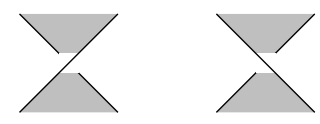

negative

Figure 1: Crossings determine the sign of the edges in the signed Tait graph.

A leaf of a graph is a 1-valent vertex; the edge incident with this vertex is called a leaf edge. Note that the first Reidemeister move either adds both a leaf edge and a leaf to the Tait graph $G$ or deletes both from $G$.

Overlaying $G$ with its plane dual $G^{*}$ (which is the Tait graph corresponding to the white regions), one obtains the overlaid Tait graph $\widehat{\Gamma}$. This graph is bipartite: its black vertex set corresponds to the intersections of an edge of $G$ with its dual edge in $G^{*}$, and its white vertex set corresponds to the vertices of both the Tait graph $G$ and its plane dual $G^{*}$. That is, $V(\widehat{\Gamma})=\left[E(G) \cap E\left(G^{*}\right)\right] \sqcup\left[V(G) \sqcup V\left(G^{*}\right)\right]$. The edges of this graph are the half-edges of both Tait graphs. A similar notion is found in work by Huggett, Moffatt, and Virdee [32].

All of the black vertices of $\widehat{\Gamma}$ are four-valent, as these correspond with vertices of the universe $U$, and all of the faces of $\widehat{\Gamma}$ are square, as these correspond to edges of the universe $U$, as in Fig. 2.

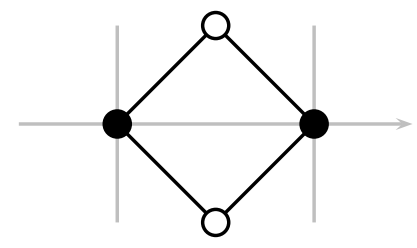

Figure 2: A square face of the overlaid Tait graph $\widehat{\Gamma}$.

In order to consider perfect matchings, delete the two starred white vertices to obtain the balanced overlaid Tait graph $\Gamma$ that is the central graph for the present paper. For more details of this construction see [16] or consider the following alternative definition.

Definition 1. The balanced overlaid Tait graph $\Gamma$ is a bipartite graph that can be obtained from a universe $U$ as follows. Let every four-valent vertex in the universe $U$ be a black 
vertex in $\Gamma$. Select two adjacent faces of $U$ and mark them $*$ by stars. Let every nonstarred face of $U$ be a white vertex in $\Gamma$. A black vertex is adjcent to a white vertex whenever the vertex and face of $U$ are incident.

Remark 2. Since $U$ is a plane graph, so is $\Gamma$. Furthermore, all faces of $\Gamma$ are square except for the infinite face. Let the boundary cycle of this infinite face be called the periphery. All black vertices not on the periphery are still four-valent. The remaining black vertices (exactly those affected by the deletion of the two starred white vertices in $\widehat{\Gamma}$ ) have valence determined by the following Periphery Proposition.

A crossing is called nugatory if there exists a circle in the projection plane meeting the diagram transversely at that crossing but not meeting the diagram at any other point. Specifically it can be easily removed by twisting some part of the diagram.

Proposition 3. Periphery Proposition. The balanced overlaid Tait graph $\Gamma$ for a diagram with no nugatory crossings has the following property: two of the black vertices on the periphery have valence two; the rest have valence three.

To make this more obvious we employ two lemmas to show that there can be no black vertices on the periphery of degree one or four.

Lemma 4. The balanced overlaid Tait graph $\Gamma$ for a diagram with no nugatory crossings has no black leaves.

Proof. Suppose by way of contradiction that there is a black leaf in $\Gamma$. This black vertex must be four-valent in the overlaid Tait graph $\widehat{\Gamma}$, and so it has three additional edges. Only two white vertices were deleted from $\widehat{\Gamma}$, and so at least two of these three edges must be incident with the same white vertex. However the black vertex corresponds to a crossing in the diagram, and so only opposite edges can be incident with the same white vertex. This results in a nugatory crossing, a contradiction.

Lemma 5. The balanced overlaid Tait graph $\Gamma$ for a diagram with no nugatory crossings has no four-valent black vertex on the periphery.

Proof. Suppose by way of contradiction that there is a four-valent black vertex $v_{1}$ on the periphery of $\Gamma$; then there are two white neighbors $u_{1}$ and $u_{2}$ of $v_{1}$ that are also neighbors of $v_{1}$ on the periphery. Since all faces of the overlaid Tait graph $\widehat{\Gamma}$ are square, there must be some black vertex $v_{2}$ such that these vertices form a square face in $\widehat{\Gamma}$ that lives in the infinite face of $\Gamma$. However, no black vertices were deleted from $\widehat{\Gamma}$ to obtain $\Gamma$, and so $v_{2}$ must be in $\Gamma$.

This can only be the case when the periphery of $\Gamma$ is itself the square with these four vertices. Here every face of $\Gamma$ is a square, so by counting the edges around each face $2|E|=4|F|$. Let $n$ be the number of black vertices; then by counting the edges around each black vertex, $|E|=4 n$. Together these facts give $|F|=2 n$.

Since this graph is a plane graph, Euler's formula gives that the number of white vertices must be $n+2$, contradicting the condition that $\Gamma$ is balanced. In fact, these properties describe $\widehat{\Gamma}$. 
Proof of the Periphery Proposition 3. Let $n_{i}$ be the number of black vertices with valence $i$; by construction $i \leqslant 4$ and by Lemma $4 i \geqslant 2$. Since the graph $\Gamma$ is balanced, $|V|=$ $2\left(n_{2}+n_{3}+n_{4}\right)$. Summing the edges around each black vertex we obtain $|E|=\sum i n_{i}=$ $2 n_{2}+3 n_{3}+4 n_{4}$. Since this is a plane graph, Euler's formula gives $|F|=2-|V|+|E|=$ $2-2 n_{2}-2 n_{3}-2 n_{4}+2 n_{2}+3 n_{3}+4 n_{4}=2+n_{3}+2 n_{4}$.

By Lemma 5 there are no four-valent black vertices on the periphery; then its length is $2\left(n_{2}+n_{3}\right)$. Summing the edges around each face we obtain $2|E|=\sum i f_{i}=4(|F|-$ 1) $+\left(2\left(n_{2}+n_{3}\right)\right)(1)=4|F|-4+2 n_{2}+2 n_{3}$. Substituting for $|F|$ as above, we obtain $n_{2}=2$.

Remark 6. Although the Periphery Proposition 3 appears itself to be a slightly unnatural restriction, it follows by the argument above that it comes directly from the more natural conditions of $\Gamma$ being plane bipartite and having black vertices of degree at most four.

The balanced overlaid Tait graph $\Gamma$ completely determines the universe $U$. Crossing information can be obtained by choosing a certain weighting on the graph.

Proposition 7. One can obtain a unique universe $U$ from the balanced overlaid Tait graph $\Gamma$.

Proof. Viewing the universe $U$ as a four-valent graph, the edges of this graph correspond to square faces of $\widehat{\Gamma}$, specifically traversing from black vertex to black vertex through the face.

Thus it is enough to show that one can uniquely produce the overlaid Tait graph $\widehat{\Gamma}$ from the balanced overlaid Tait graph $\Gamma$.

Identify the periphery with the unit circle such that the two black vertices on it that have valence two are at position 1 and -1 . Add two new white vertices at $2 i$ and $-2 i$ that are adjacent to both of these black vertices. Furthermore, the white vertex at $2 i$ (or $-2 i$ ) is adjacent to every black vertex on the upper hemisphere (or lower hemisphere, respectively) of the periphery.

One can see this is unique because exactly two white vertices need to be added in a planar way adjacent to the two black two-valent vertices on the periphery, and the rest of the black vertices on the periphery are three-valent.

Thus this is the graph $\Gamma$ that we will consider, given a specific diagram $D$ with two specified adjacent starred regions for a specific knot $K$.

\section{The graph of perfect matchings}

We now construct the graph $\mathcal{G}$ of perfect matchings of the bipartite graph $\Gamma$ above. Unless otherwise specified, we assume $\Gamma$ has the properties as mentioned above and can be obtained from a diagram $D$ of a knot $K$.

We formally take the vertices of $\mathcal{G}$ to be the perfect matchings of $\Gamma$, although the reader may choose to interpret these vertices instead as states of a universe $U$ as in [35] and [1]. An edge in $\mathcal{G}$ corresponds to a flip move of perfect matchings, that is, where all 
but two of the edges of each perfect matching agree, and these four edges create a square face.

The reader may instead consider $\mathcal{G}$ as the clock lattice $\mathcal{L}$ constructed in [35] and [1]. Here the edges are directed according to the clock move as in Figure 3. Given the square face from the flip move as a cycle in the plane oriented counterclockwise, the perfect matching whose edges on this oriented cycle go from white to black is the tail of the directed edge, and the one that goes from black to white is the head.

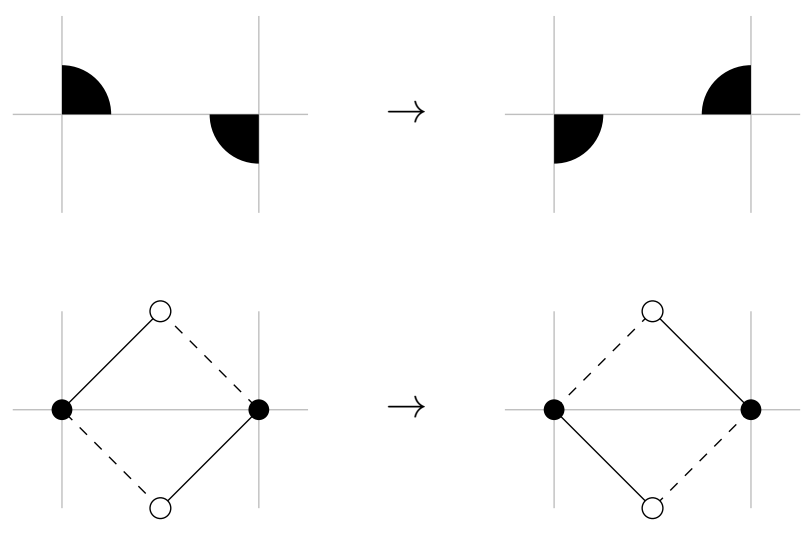

Figure 3: The clock move.

Kauffman proves the following for the clock lattice of a universe.

Theorem 8. [35, Clock Theorem 2.5.] Let $U$ be a universe and $\delta$ the set of states of $U$ for a given choice of adjacent fixed stars. Then $\delta$ has a unique clocked state and a unique counterclocked state. Any state in $\delta$ can be reached from the clocked (counterclocked) state by a series of clockwise (counterclockwise) moves. Hence any two states in $\delta$ are connected by a series of state transpositions.

At the end of the proof before Section 3, Kauffman also shows that the collection of states is a lattice. As explicitly asserted by Gilmer and Litherland [28, The Clock Theorem], this is in fact a graded distributive lattice.

Denote the unique minimum by $\widehat{0}$ and the unique maximum by $\widehat{1}$ of the connected lattice $\mathcal{L}$; these are also called the clocked and counterclocked states, respectively, in the graph $\mathcal{G}$. Let $h$ be the height of the lattice.

The diameter of a graph is the maximum of the shortest distance, or number of edges, between any two vertices taken over all pairs of vertices.

Proposition 9. The height h of the clock lattice $\mathcal{L}$ is indeed the diameter of the graph $\mathcal{G}$.

Proof. Since $h$ is the distance between $\widehat{0}$ and $\widehat{1}$, it is enough to show that the distance between any other two elements is no greater than $h$.

Choose any two elements $x$ and $y$ with heights $h(x)$ and $h(y)$, respectively. Then there are always at least two paths between $x$ and $y$ : one through $\widehat{0}$ and another through $\widehat{1}$. 
These two paths have distances $h(x)+h(y)$ and $2 h-(h(x)+h(y))$. Thus if $h(x)+h(y)>h$, the second path is less than $h$.

We may call $h+1$ the clock number of the diagram $p(D)$ for a diagram $D$ with chosen starred regions. Note that this number is dependent on the actual diagram of the knot given and is not invariant over all diagrams.

To turn this into a knot invariant, Abe [1] takes the minimum of $p(D)$ over all diagrams $D$ of a knot $K$ and calls this the clock number $p(K)$ of the knot. The two main theorems of this work by Abe are that $p(K) \geqslant c(K)$, the crossing number of the knot, with equality when $K$ is a two-bridge knot.

The two-bridge knots are well-understood as the closures of rational tangles and can be presented as in Figure 4, where the two loose ends are joined and where the non-zero integer $a_{i}$ represents the number of positive or negative twists in each region.

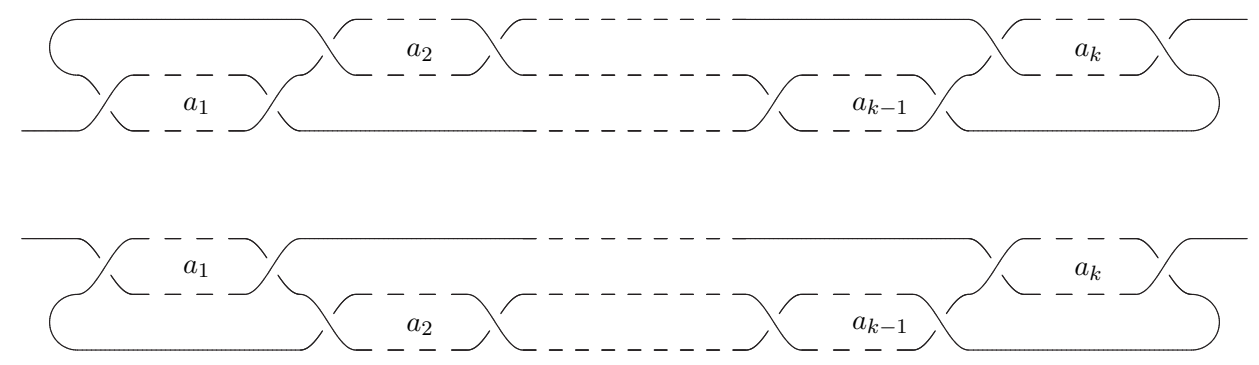

Figure 4: Conway forms for two-bridge knots, as taken from [40], once the loose ends have been joined.

A bridge is one of the arcs in a diagram; thus it consists only of over-crossings. The bridge index $\operatorname{br}(K)$ of a knot $K$ is the minimum number of disjoint bridges which together include all over-crossings, considering all diagrams. An equivalent definition for the bridge index uses a Morse function and counts the number of local maxima of the knot, after taking the minimum over all diagrams. By turning each of the diagrams of Figure 4 on its side, it is easy to see the two local maxima, once the loose ends have been joined.

\section{Notions from graph theory}

\subsection{Connectivity}

The unordered pair $\{A, B\}$ of vertex subsets is a $k$-separation of a graph if $A \cup B$ gives the entire vertex set, $|A \cap B|=k$, and the graph has no edge between $A \backslash B$ and $B \backslash A$. Equivalently a subset $X$ of vertices and edges is said to separate two vertex sets $A$ and $B$ if every $A-B$ path in the graph contains a vertex or edge from $X$. The following theorem will be useful below: 
Theorem 10. [22, Theorem 3.3.1. (Menger 1927)] Let $G=(V, E)$ be a graph and let $A, B \subseteq V$. Then the minimum number of vertices separating $A$ from $B$ in $G$ is equal to the maximum number of disjoint $A-B$ paths in $G$.

A knot $K$ is called prime in standard terminology if when it is written as a connect sum $K=K_{1} \# K_{2}$, either $K_{1}$ or $K_{2}$ must be the unknot. Below we say that a knot diagram $D$ is prime-like if the diagram cannot be written as a conncect sum of diagrams $D=D_{1} \# D_{2}$ where there are crossings in both $D_{1}$ and $D_{2}$.

Lemma 11. The following are equivalent for a diagram $D$ for a knot $K$ with no nugatory crossings:

1. the diagram $D$ is not prime-like;

2. the Tait graph $G$ has a cutvertex;

3. the dual Tait graph $G^{*}$ has a cutvertex; and

4. the overlaid Tait graph $\widehat{\Gamma}$ has a 2-separation: namely the two cutvertices of $G$ and $G^{*}$ above separate the graph.

Furthermore, there is an arc of the knot diagram incident with the regions associated to both cutvertices.

Proof. The implication $(1) \Rightarrow(2)$ holds by Figure 5 . The converse also holds because a circle around one component of the graph meeting only at the cutvertex is the same circle that encloses one of the diagrams in the connect sum.

The implication $(2) \Leftrightarrow(3)$ holds by Figure 5 and the fact that the Tait graph is unchanged by ambient isotopy of the knot diagram on a sphere.

The implication $(2) \Leftrightarrow(4)$ holds because the circle from above is also the circle that encloses one of the sets in the 2-separation of $\widehat{\Gamma}$ meeting it only at the two cutvertices of $G$ and $G^{*}$.

Lastly, if the two cutvertices were not incident with a single arc of the knot diagram, then there would be another region in between them, violating all of the above.

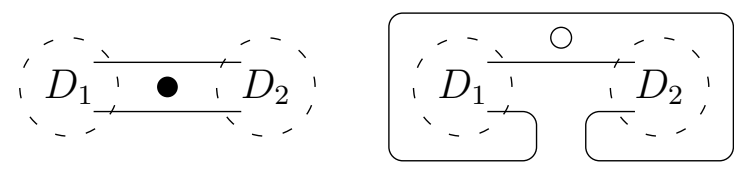

Figure 5: Cutvertices in $G$ and $G^{*}$.

Remark 12 . Observe that since the two cutvertices of $G$ and $G^{*}$ separate $\widehat{\Gamma}$, the deletion of both of these vertices makes $\Gamma$ disconnected while the deletion of one of these vertices makes $\Gamma$ 1-connected. 
Proposition 13. The Tait graph $G$ for a prime-like knot diagram with no nugatory crossings is 2-connected. This also holds for its dual $G^{*}$.

Proof. The Tait graph $G$ must be connected because the diagram is for a knot with a single component. By Lemma 11 there can be no cutvertices.

The result that $\Gamma$ is 2-connected is Proposition 19 at the end of the next subsection. In order to show this, we introduce the following possibly unfamiliar idea.

\section{$4.2 \quad$ Elementary graphs}

We make specific mention of the following definitions, as they may be unfamiliar to many readers.

Definition 14. An edge of any graph is allowed if it lies in some perfect matching of the graph and forbidden otherwise. A graph is elementary if its allowed edges form a connected subgraph of the graph.

Recall that a vertex covering is a subset of vertices such that every edge has at least one endpoint in the vertex subset. Denote by $\nu(X)$ the set of neighbors of a subset $X$ of vertices. Let $K_{2}$ be the complete graph on two vertices: that is, a single edge.

Theorem 15. [49, Theorem 4.1.1.] Given a bipartite graph with a bipartition $(U, W)$ of the vertex set, the following are equivalent:

1. the graph is elementary;

2. the graph has exactly two vertex coverings, namely $U$ and $W$;

3. $|U|=|W|$ and for every non-empty proper subset $X$ of $U,|\nu(X)| \geqslant|X|+1$;

4. the graph is $K_{2}$, or there are at least four vertices and for any $u \in U, w \in W$, the graph with these two vertices deleted has a perfect matching; and

5. the graph is connected and every edge is allowed.

In order to prove the next main theorem for this subsection, one must remove any nugatory crossings from the knot diagram before taking the associated balanced overlaid Tait graph.

Theorem 16. The balanced overlaid Tait graph $\Gamma$ for a prime-like knot diagram with no nugatory crossings is an elementary graph.

Proof. Consider some edge $\varepsilon \in E(\Gamma)$ in the balanced overlaid Tait graph. By Theorem 15 (5) we must show that this edge is allowed, that is, that it belongs to some perfect matching of $\Gamma$. Note that $\varepsilon$ is also an edge in the overlaid Tait graph $\widehat{\Gamma}$ before two white vertices are deleted. 


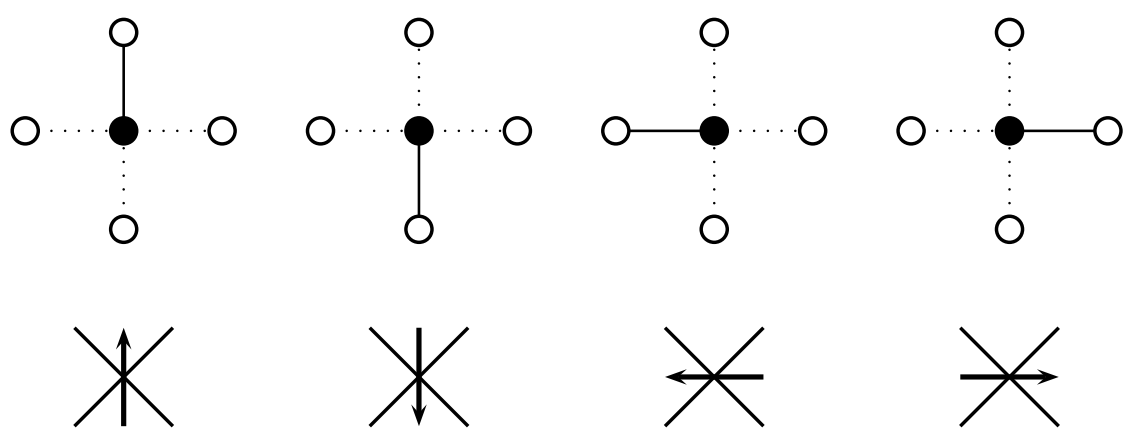

Figure 6: The correspondence between edges $\varepsilon$ in the overlaid Tait graph $\widehat{\Gamma}$ and directed edges $e$ in the (directed) Tait graph $G$.

According to [16, Proposition 4.8], there is a bijection between perfect matchings of the balanced overlaid Tait graph $\Gamma$ and rooted spanning trees of one of the Tait graphs $G$ or its dual $G^{*}$. Furthermore, this work gives a bijection between the edge $\varepsilon \in E(\Gamma)$ and a directed edge $\vec{e} \in E(G) \cup E\left(G^{*}\right)$ in one of the two Tait graphs, as in Figure 6 .

Thus it is enough to show that the directed edge $\vec{e} \in E(G) \cup E\left(G^{*}\right)$ belongs to some rooted spanning tree of either $G$ or its dual $G^{*}$. If there are no nugatory crossings, then this edge cannot be a loop or a bridge. So the undirected edge $e$ belongs to some un-rooted spanning tree.

To see that both orientations of this edge occur, it is enough to show that there is a cycle containing both $e$ and the starred vertex where $e$ is not incident to the starred vertex. Then an edge can be removed from either end of the cycle to produce either orientation on the remaining edges of the cycle. Observe that if $e$ is incident with the starred vertex then the edge of $\widehat{\Gamma}$ corresponding to the wrong orientation of the edge of $G$ was deleted to produce $\Gamma$.

Since $e$ is not a loop or bridge, it belongs to some cycle $C$. Since by Proposition 13 the Tait graph $G$ is 2-connected, there can be no 1-separation, and so a separating set must be of size at least two. Apply Menger's Theorem 10 with $A$ being the neighbors of the starred vertex and $B$ being the cycle $C$; then there must be two disjoint $A$ - $B$ paths $P_{1}$ and $P_{2}$ in $G$. In particular, the endpoints in the cycle $C$ cannot be the same, and so these endpoints partition $C$ into two paths, one of which contains $e$, say $P_{3}$. Then $P_{1} \cup P_{2} \cup P_{3}$ is a cycle containing both $C$ and the starred vertex, as desired.

In particular, this will show that $\Gamma$ for a prime-like knot diagram with no nugatory crossings is 2-connected. First we need another possibly unfamiliar definition.

Definition 17. A graph $\Gamma$ is said to be $n$-extendable if it is connected, has a set of $n$ independent edges, and every set of $n$ independent edges in $\Gamma$ extends to (i.e. is a subset of) a perfect matching of $\Gamma$.

Then by Theorem 15 (5) an elementary bipartite graph is 1-extendable.

Lemma 18. [52, Lemma 3.1] Every 1-extendable graph (that is not $K_{2}$ ) is 2-connected. 
This gives the desired result.

Proposition 19. The balanced overlaid Tait graph $\Gamma$ for a prime-like knot diagram with no nugatory crossings is 2-connected.

\section{$5 \quad$ Partitioning the vertex set into leaves and cycles}

The main idea of this section is to partition the vertices of the graph $\Gamma=\Gamma_{1}$ into leaves $\ell \in L^{V}$ and cycles $C_{i}$, denoting by $\Gamma_{i}$ the interior graph within and including the cycle $C_{i}$. The proof of the main theorem of this section, Theorem 27, will involve two induction steps: first the existance of the next cycle and second that it satisfies the Periphery Proposition 3.

In the simplest cases, one need only delete the periphery to obtain the next interior graph, whose periphery is the next cycle. This can be seen in Figure 7 for $\Gamma$ obtained from a diagram of the knot $K 11 n 157$ : the cycle $C_{1}$ in blue serves as the periphery of the interior graph $\Gamma=\Gamma_{1}$ shaded in blue; deleting this cycle results in the new interior graph $\Gamma_{2}$ shaded in red, whose periphery is the cycle $C_{2}$ in red. What is left after $C_{2}$ is deleted is a leaf edge in green.

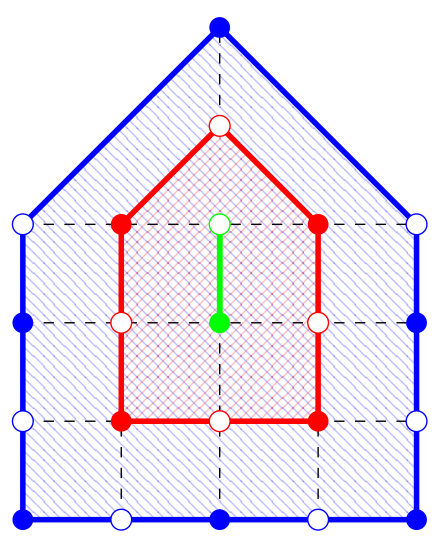

Figure 7: The desired partition of the vertices of $\Gamma$ obtained from a diagram for the knot $K 11 n 157$ : the cycle $C_{1}$ in blue with its interior graph $\Gamma=\Gamma_{1}$ shaded in blue, the cycle $C_{2}$ in red with its interior graph $\Gamma_{2}$ shaded in red, and the leaf edge in green.

These cycles $C_{i}$ emerge when the symmetric difference is taken of the clocked and counterclocked states $\widehat{0}$ and $\widehat{1}$ of Kauffman's clock lattice!

\subsection{Some final useful tools}

Again we make specific mention of the following definitions, as they may be unfamiliar to many readers. 
Definition 20. A cycle (or a path) of $\Gamma$ is called $\mu$-alternating if the edges of the cycle (or path) appear alternately in the perfect matching $\mu$ and $E(\Gamma) \backslash \mu$. A face of a 2-connected plane bipartite graph is called resonant if its boundary is a $\mu$-alternating cycle for some perfect matching $\mu$.

One can think of the perfect matching $\mu$ lying along the $\mu$-alternating cycle (or path) in the fashion of a dashed line. More frequently than not, a cycle (or path) will not be $\mu$-alternating, as there are other edges to choose from incident with each vertex of this cycle (or path).

The next theorem is the reason we will be concerned with proving graphs are 2connected and elementary.

Theorem 21. [57, Theorem 2.4.] Given a plane bipartite graph with more than two vertices, each face (including the infinite face) is resonant if and only if the graph is elementary.

Denote by $q(G)$ the number of components of a graph $G$ with an odd number of vertices. This theorem shall be used below.

Theorem 22. [22, Theorem 2.2.1. (Tutte 1947)] A graph $G$ has a perfect matching if and only if the number of odd components $q(G-S) \leqslant|S|$ for all subsets $S \subseteq V(G)$.

We introduce the following two operations on cutvertices that will be used in the next subsection.

Definition 23. "Pruning" leaf edges. Suppose a cutvertex is incident with a leaf edge: the edge incident with a one-valent vertex. The operation called "pruning" a leaf edge from a graph will mean deleting all edges adjacent to the leaf edge. A graph is "pruned" when all of its leaf edges have been pruned; this operation must be done recursively if pruning a leaf edge results in a new leaf edge. Collect all of the leaves and leaf edges pruned in this way in the sets $L^{V}$ and $L^{E}$, respectively. Note that here two vertices are deleted at a time, one from each vertex set if the graph is bipartite as below.

Definition 24. "Breaking" cutvertices. Suppose the deletion of a cutvertex would result in more than one component, each of which contains a cycle (when including the cutvertex). Also suppose that there is exactly one component that has an odd number of vertices (not including the cutvertex). The operation called "breaking" the cutvertex from a graph will mean deleting all edges incident with the cutvertex except for those in the odd component. A graph is "broken" if there are no more cutvertices.

Remark 25. These operations are non-standard in graph theory. In other contexts, breaking a cutvertex might produce two graphs that both contain the cutvertex. This name perhaps comes from the operation of breaking "handcuffs", where the tight handcuff graph is two cycles with a single vertex in common and the loose handcuff graph is two cycles connected by a path. 
Finally there is an argument that, while trivial, will occur in several places in the proof in the next subsection. In particular, this will be used when a construction allows for the repeated use of new leaf edges rather than stopping at one of the other cases.

Lemma 26. Finite Leaf Edge Lemma. The can be only a finite number of leaf edges.

Proof. There is only a finite number of vertices because there is only a finite number of crossings because the knot is tame. Wild knots are not considered in this work.

\subsection{Main Construction}

Next is the important construction:

Theorem 27. Consider the balanced overlaid Tait graph $\Gamma$ for a prime-like knot diagram with no nugatory crossings. Then the vertices can be partitioned into leaves $\ell \in L^{V}$ and cycles $C_{i}$, where each cycle $C_{i}$ satisfies the Periphery Proposition 3 and where each interior graph $\Gamma_{i}$ is elementary and 2-connected.

By Theorem 21, these two properties of $\Gamma_{i}$ allow us to use induction below.

Corollary 28. Every face (and specifically the periphery $C_{i}$ ) of each interior graph $\Gamma_{i}$ is resonant.

Proof of Theorem 27. The periphery $C=C_{1}$ on the infinte face satisfies the Periphery Proposition 3, and $\Gamma=\Gamma_{1}$ is elementary by Theorem 16 and 2-connected by Proposition 19. The proof will proceed by constructing the next $C_{i}$ assuming that for all $j<i$ all previous $C_{j}$ already satisfy the Periphery Proposition 3 and that all previous $\Gamma_{j}$ are elementary and 2-connected.

Thus each previous periphery $C_{j}$ is resonant, and so there is a perfect matching $\mu$ such that $C_{j}$ is $\mu$-alternating. This ensures that when the periphery $C_{j}$ is deleted from the interior graph $\Gamma_{j}$, the remaining graph still has some perfect matching: specifically $\mu$ restricted to $\Gamma_{j} \backslash\left\{C_{j}\right\}$.

\subsection{Construction}

Delete all the edges incident with vertices in the cycle $C_{i-1}$ from the graph $\Gamma_{i-1}$ to obtain a new graph $\Gamma_{i}^{\prime}$, and consider the edges $C_{i}^{\prime}$ on the new periphery. If $C_{i}^{\prime}$ has several components, treat each $C_{i}^{\prime}, C_{i+1}^{\prime}, \ldots$ separately. If some component $C_{i}^{\prime}$ is indeed a single cycle with no cutvertices, then set $C_{i}=C_{i}^{\prime}$ and $\Gamma_{i}=\Gamma_{i}^{\prime}$.

Otherwise there is some cutvertex, possibly on a leaf edge. One can employ some sequence of "pruning" leaf edges and "breaking" cutvertices as follows. It is important to note here that when there are several cutvertices, the leaves and cycles must be connected in a tree-like way, and so one can start by the outermost edges of this tree.

From pruning leaf edges one obtains a new set of outer edges $C_{i}^{\prime \prime}$ of the new interior graph $\Gamma_{i}^{\prime \prime}$. If this is indeed a single cycle with no cutvertices, then set $C_{i}=C_{i}^{\prime \prime}$ and 
$\Gamma_{i}=\Gamma_{i}^{\prime \prime}$. Collect the leaves and leaf edges pruned in this way in the sets $L_{i-1}^{V}$ and $L_{i-1}^{E}$, respectively. Note that here two vertices are deleted at a time, preserving the property that the interior graph $\Gamma_{i}^{\prime \prime}$ has equal-sized vertex sets.

In order to break additional cutvertices, one must show that there is exactly one component that has an odd number of vertices when the cutvertex is deleted from $\Gamma_{i}^{\prime \prime}$.

Lemma 29. Suppose the graph $\Gamma_{i}^{\prime}$ (or $\Gamma_{i}^{\prime \prime}$ ) has a cutvertex. Then after deleting this cutvertex, exactly one connected component has an odd number of vertices.

Proof. First observe by Theorem 21 on the 2-connected elementary interior graph $\Gamma_{i-1}$ that there must be a perfect matching $\mu$ such that the periphery $C_{i-1}$ of $\Gamma_{i-1}$ is $\mu$ alternating. This perfect matching $\mu$ must include the leaf edges deleted above.

Now consider the graph $\Gamma_{i}^{\prime}$ (or $\Gamma_{i}^{\prime \prime}$ ) with the subset $S$ being just the cutvertex as in the statement of Theorem 22. Then the number of odd components is at most one. Because the graph is bipartite, the number of odd components must be odd, so this number is indeed one.

From breaking the cutvertex one obtains several components with peripheries $C_{i}^{\prime \prime \prime}$, $C_{i+1}^{\prime \prime \prime}, \ldots$ of new interior graphs $\Gamma_{i}^{\prime \prime \prime}, \Gamma_{i+1}^{\prime \prime \prime}, \ldots$ for each of the components. Repeat this process until each of these is indeed a single cycle with no cutvertices, then set $C_{j}=C_{j}^{\prime \prime \prime}$ and $\Gamma_{j}=\Gamma_{j}^{\prime \prime \prime}$ for $j \geqslant i$.

\subsection{Cycles satisfy the Periphery Proposition 3}

After a brief useful lemma, the proof continues by showing that $C_{i}$ satisfies the Periphery Proposition 3.

Lemma 30. Inchworm Lemma. A square face $f$ in $\Gamma_{i-1}$ cannot have exactly one edge on $C_{i}$ while its opposite edge is a leaf edge $\ell \in L_{i-1}^{E}$; otherwise $C_{i}$ could have been extended to include $\ell$ and $f$ would be a part of $\Gamma_{i}$.

Proof. This follows from the construction of the cycle $C_{i}$ in $\Gamma_{i-1}$.

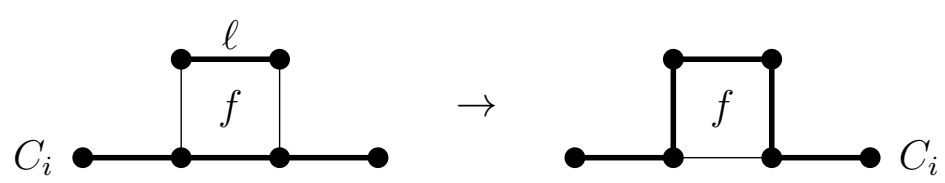

Figure 8: The situation on the left cannot occur by the Inchworm Lemma 30. The vertex sets are undistinguished.

Lemma 31. Periphery Proposition Lemma. The periphery $C_{i}$ of the internal graph $\Gamma_{i}$ satisfies the Periphery Proposition 3. 
Proof. In order to apply the same proof of the Periphery Proposition 3, we must ensure that only two-valent and three-valent black vertices can be on $C_{i}$. It is clear that there are no black leaves by construction. Thus together with Sublemma 32 the proof can be applied in this case.

Sublemma 32. There can be no four-valent black vertices on the cycle $C_{i}$.

Proof. Suppose by way of contradiction that there is a four-valent black vertex $v_{1}$ on the periphery $C_{i}$ of the interior graph $\Gamma_{i}$; note that $v_{1}$ cannot be a cutvertex as these were broken above. Then there are two white neighbors $u_{1}$ and $u_{2}$ of $v_{1}$ that are also neighbors of $v_{1}$ on the cycle $C_{i}$. Then $u_{1}, v_{1}, u_{2}$ must form a square face with some other black vertex $v_{2}$ in the interior graph $\Gamma_{i-1}$ but outside of the interior graph $\Gamma_{i}$. We may assume by induction hypothesis that $v_{2}$ cannot be on $C_{i-1}$, or else it would be four-valent there. Also this black vertex cannot be on $C_{i}$ or the cycle would extend through to include this face.

Then the black vertex $v_{2}$ must either be on a leaf edge or on another interior cycle $C_{i+1}$. If it is on a leaf edge with white vertex $u_{3}$, then with either $u_{1}$ or $u_{2}$ this leaf edge forms a square face with some other black vertex $v_{3}$, which also cannot be on $C_{i-1}$ as above or on $C_{i}$ by the Inchworm Lemma 30. By the Finite Leaf Edge Lemma 26, this process eventually terminates, as so we may assume that our original other black vertex $v_{2}$ is on another interior cycle $C_{i+1}$.

Then $v_{2}$ has two white neighbors $u_{3}$ and $u_{4}$ on the cycle $C_{i+1}$. It can have no other neighbors since a black vertex is at most four-valent. Then $u_{1}, v_{2}$, and $u_{3}$ are on a square face in $\Gamma_{i-1}$ that also contains some black vertex $v_{3}$; note additionally that $u_{2}, v_{2}$, and $u_{4}$ are on a square face in $\Gamma_{i-1}$ that also contains some black vertex $v_{4}$, but we need only consider one of these. Following a similar argument as above, this black vertex cannot appear on $C_{i-1}$ as it would be four-valent or $C_{i}$ or $C_{i+1}$ as this would alter the cycle structure. It may appear on a leaf edge, but by the Finite Leaf Edge Lemma 26 this process eventually terminates. The only remaining option is for $v_{3}$ to appear on a new interior cycle $C_{i+2}$.

However, this situation again forces two new black vertices that must be handled according to the above arguments. What is more is that no new black vertex created can appear on a previous interior cycle, as it would alter the cycle structure. Thus ultimately all new interior cycles and leaf edges are exhausted and there are no remaining options for the black vertex, a contradiction.

\subsection{Each interior graph is 2-connected and elementary}

The interior graph $\Gamma_{i}$ is 2-connected by construction: it is connected because each component was considered separately, and all cutvertices were removed after "pruning" leaf edges and "breaking" cutvertices.

To prove that the interior graph $\Gamma_{i}$ is elementary, we apply the proof of Theorem 16 to the interior graph $\Gamma_{i}$ after it has been turned into a diagram $D_{i}$ by Proposition 7 . Note 
that this diagram is prime-like with no nugatory crossings by construction. It does not matter if the diagram represents a knot or a link with several components.

Remark 33. As inferred by the end of the proof, the interior graphs $\Gamma_{i}$ are in fact themselves balanced overlaid Tait graphs.

Question 34. Can the balanced overlaid Tait graphs $\Gamma_{i}$ be related to each other in an analogous way to tangles and sub-tangles?

\section{Reduction moves and black two-valent vertices}

The purpose of this section is to highlight several local moves that simplify the structure of the interior graph $\Gamma_{i-1}$. These will be used to prove the main result, Theorem 56, on the height of Kauffman's clock lattice.

The first reduction move can be used to simplify each interior graph $\Gamma_{i-1}$ by removing leaf edges without altering the structure of the cycles.

Proposition 35. Leaf Edge Reduction Proposition. Suppose the interior graph $\Gamma_{i-1}$ obtained from a knot diagram that has no nugatory crossings contains some alternating path of leaf edges. Then this graph can be reduced to one without the leaf edges by a local move as depicted in Figure 9.

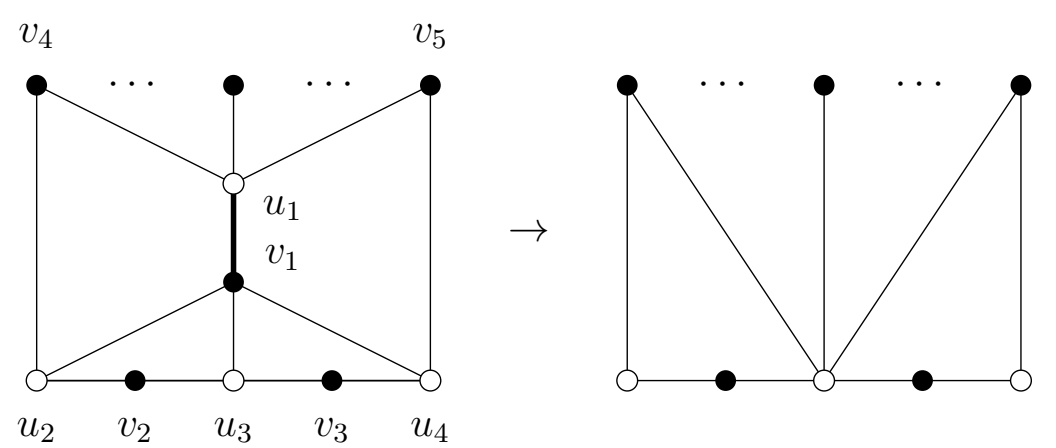

Figure 9: The local operation of reduction on a single leaf edge.

Proof. It is enough to show that the square faces depicted in the left hand side of Figure 9 arise. Then they can be reduced to those that appear on the right hand side because the graph is still bipartite, all faces are still square, and the valence of the black vertices does not change.

Note that any leaf edges mentioned here are not actual leaf edges in $\Gamma$ but are those edges in $L^{E}$ produced in the partition of the main construction Theorem 27 .

Consider the black vertex $v_{1}$ on the leaf edge along with its white neighbors $u_{1}, u_{2}$, $u_{3}$, and $u_{4}$ as in the left hand side of Figure 9. Observe that these four neighbors must 
be distinct: identifying any two forms either a bigon or a nugatory crossing that would be in both $\Gamma_{i-1}$ as well as $\Gamma$. Then there is a square face containing $u_{2}, v_{1}$, and $u_{3}$ that must also contain some black vertex $v_{2}$ and a square face containing $u_{3}, v_{1}$, and $u_{4}$ that must also contain some black vertex $v_{3}$.

If $v_{2}=v_{3}$ are not distinct, this produces another leaf edge as in Figure 10, and there are again two black vertices to be considered. Continue this process creating the alternating path of leaf edges, ending with two distinct black vertices because of the Finite Leaf Edge Lemma 26. This creates the square faces depicted in the lower parts of the images on the left hand side of Figures 9 and 10.

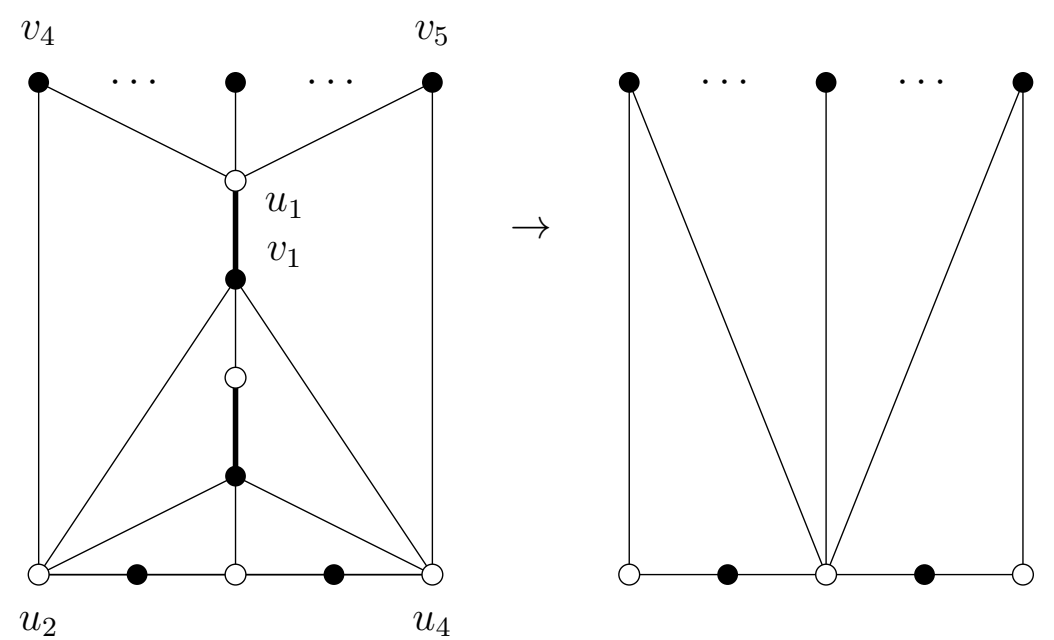

Figure 10: The local operation of reductions on a path of leaf edges.

To see the square faces in the upper parts of these images, observe that there is a square face containing $u_{1}, v_{1}$, and $u_{2}$ that must also contain some black vertex $v_{4}$ and a square face containing $u_{1}, v_{1}$, and $u_{4}$ that must also contain some black vertex $v_{5}$. Even if $v_{4}=v_{5}$ are not distinct, this move can be performed.

Remark 36. A generalized version of this reduction move can apply to leaf edges alternating on some tree by starting at one-valent white vertices on this tree, as in Figure 10 .

Remark 37. This Leaf Edge Reduction Proposition move is in fact a number of smoothings on a twist region of the knot diagram, where the number of crossings in the twist region is given by the number of leaf edges in the path.

Proposition 38. Simply Connected Region Reduction Proposition. Let $H$ be an induced subgraph of the interior graph $\Gamma_{i-1}$ with all its vertices on $C_{i-1}$ such that all but one of the edges of the periphery of $H$ are on $C_{i-1}$. Then there is a two-valent black vertex of $C_{i-1} \subset \Gamma_{i-1}$ in $H$, and $H$ can be deleted from $\Gamma_{i-1}$ by a local move without changing the structure of interior cycles. 
Proof. Consider the two vertices $v_{1}$ and $u_{1}$ on the edge $e$ not on $C_{i-1}$. By the Periphery Proposition Lemma 31 the black vertex $v_{1}$ must be three-valent on $C_{i-1}$ and so has a white neighbor $u_{2}$ on $H$. Then there is a square face in $H$ containing $u_{1}, v_{1}, u_{2}$, and some black vertex $v_{2}$, which must also be on $C_{i-1}$, as a neighbor of either $u_{1}$ or $u_{2}$. Without loss of generality we may say it is $u_{1}$; then there is an edge $u_{2} v_{2}$ of this square face that encloses a new induced subgraph $H^{\prime}$ together with a path on $C_{i-1}$ that has one fewer square face.

In this way a simply connected induced subgraph $H$ can be "unstacked" until there is only one face left with all four vertices on $C_{i-1}$. It is clear then that this gives a two-valent black vertex on $\Gamma_{i-1}$. When all faces of $H$ have been deleted, the original black vertex $v_{1}$ becomes two-valent in the new $\Gamma_{i-1}$.

Remark 39. While the Leaf Edge Reduction Proposition 35 works globally, one cannot employ the Simply Connected Region Reduction Proposition 38 in $\Gamma_{i-1}$ while considering the larger interior graph $\Gamma_{i-2}$.

Since Theorem 27 gives two black two-valent vertices on each cycle $C_{i}$, we will investigate further these black vertices.

Proposition 40. Stacking Proposition. A black two-valent vertex on $\Gamma_{i-1}$ produces one on $\Gamma_{i}$.

Proof. First use the Leaf Edge Reduction Proposition 35 to remove any leaf edges.

Now consider one of the two black two-valent vertices $v_{1}$ in $C_{i-1}$ and its two white neighboring vertices $u_{1}, u_{2}$ also in $C_{i-1}$. Then the square face on the interior graph $\Gamma_{i-1}$ containing $u_{1}, v_{1}$, and $u_{2}$ has one additional black vertex $v_{2}$.

If $v_{2}$ is not in $C_{i-1}$, then it is a two-valent vertex in some interior cycle $C_{i}$ as in Figure $11(\mathrm{~A})$.

(A)

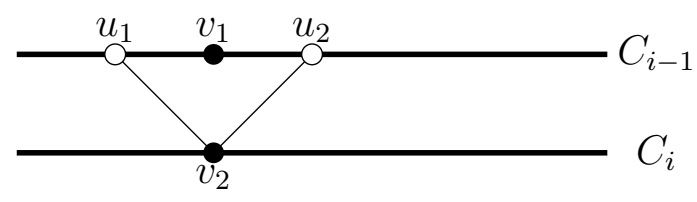

$(B)$

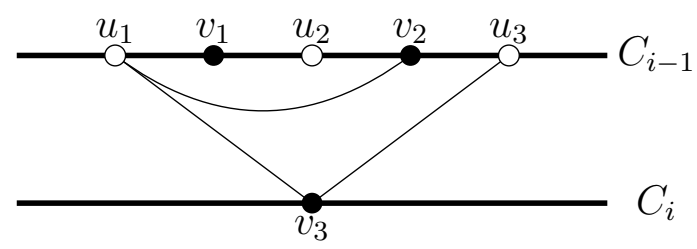

Figure 11: The two-valent vertex $v_{1}$ in $C_{i-1}$ producing another two-valent vertex in $C_{i}$.

If $v_{2}$ is indeed in $C_{i-1}$, then at least one of the two edges to $v_{2}$ from the $u_{1}, u_{2}$ must be in $C_{i-1}$, as well, in order to keep $v_{2}$ at most three-valent in $\Gamma_{i-1}$. When both of these 
edges belong to $C_{i-1}$, the graph $\Gamma_{i-1}=\Gamma_{k}$ is just a square, and there is no $C_{i}$. Supposing the edge $u_{1} v_{2}$ is not in $C_{i-1}$, we obtain the scenario depicted at the top of Figure $11(\mathrm{~B})$.

In this way more square faces may be stacked on top of each other, each pair meeting at a single edge, with all vertices appearing on $C_{i-1}$.

The outer two white vertices surrounding all the three-valent black vertices must share a common neighbor, say $v_{3}$. If $v_{3}$ is in $C_{i-1}$, as well, it is the other two-valent vertex in $C_{i-1}$, the graph $\Gamma_{i-1}$ is just these stacked squares, and there is no $C_{i}$. Otherwise $v_{3}$ is a two-valent vertex in $C_{i}$ as in the lower portion of Figure 11 (B).

Thus one can see directly how two black two-valent vertices on interior cycles arise from those on $C_{i-1}$.

Remark 41. It may happen, however, that the two black two-valent vertices produced in the proof above occur on two separate cycles $C_{i}$ and $C_{i+1}$.

Following this last remark, additional single black two-valent vertices arise when more than one connected component appears after deleting the periphery. This is handled by the following proposition.

Proposition 42. Accordion Proposition. Suppose that when $C_{i-1}$ is deleted from $\Gamma_{i-1}$ it results in two components, each containing cycles after pruning leaf edges and breaking cutvertices. Let $C_{i}$ and $C_{i+1}$ be the two cycles, one in each component, that are closest to each other using the usual notion of distance on the graph. Then this results in an extra pair of black two-valent vertices, one on each of $C_{i}$ and $C_{i+1}$.

As in Figure 12, the two interior cycles $C_{i}$ and $C_{i+1}$ appear as handles of the so-called accordion.

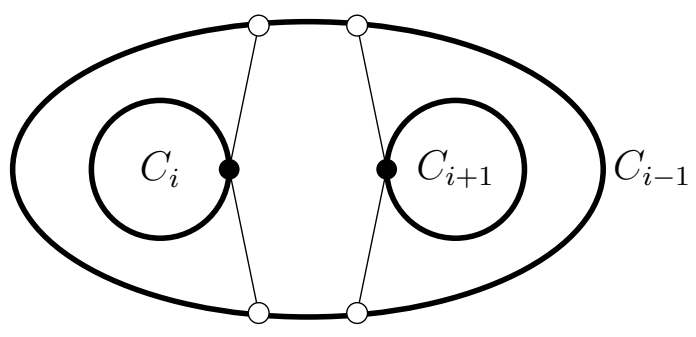

Figure 12: An accordion arises when $C_{i-1}$ is deleted from $\Gamma_{i-1}$ leaving two components.

Proof. First use the Leaf Edge Reduction Proposition 35 to remove the leaf edges. Observe that this does not change the structure of the cycles in consideration here.

Because the interior graph $\Gamma_{i-1}$ is 2-connected, Menger's Theorem 10 states that there are at least two internally disjoint paths from $C_{i}$ to $C_{i+1}$. Specifically, one can choose two disjoint paths whose interior vertices are all on $C_{i-1}$, one on each side.

Choose two such paths, each with minimal distance. Observe that this distance must be at least two; otherwise $C_{i}$ and $C_{i+1}$ are connected by an edge in $\Gamma_{i-1} \backslash C_{i-1}$, contradicting the assumption. 
These two paths form a cycle that is the periphery of an induced subgraph $H$ with no other vertices in it, since the Leaf Edge Reduction Proposition has removed leaf edges and since $C_{i}$ and $C_{i+1}$ are nearest to each other by distance. The periphery of $H$ is composed of some possibly trivial path on $C_{i-1}$, an edge from $C_{i-1}$ to $C_{i}$, some possibly trivial path on $C_{i}$, another edge from $C_{i}$ to $C_{i-1}$, another possibly trivial path on $C_{i-1}$, an edge from $C_{i-1}$ to $C_{i+1}$, some possibly trivial path on $C_{i+1}$, and another edge from $C_{i+1}$ to $C_{i-1}$ as in Figure 13.

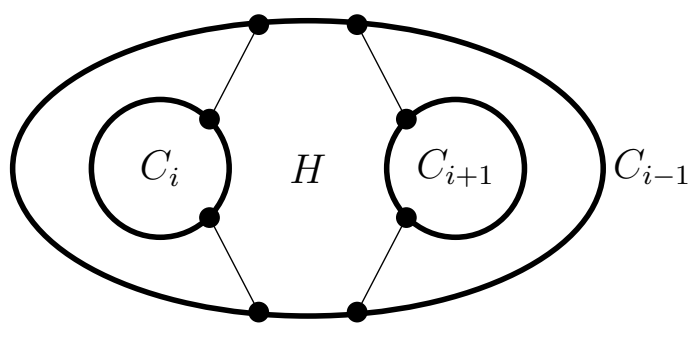

Figure 13: A general set-up used in the proof of the Accordion Proposition 42, with the vertex sets undistinguished.

Next we show that the periphery of the induced subgraph $H$ satisfies the Periphery Proposition 3. As in the Periphery Proposition Lemma 31, in order to apply the same proof of the Periphery Proposition 3, we must ensure that only two-valent and three-valent black vertices can be on the periphery of $H$. It is clear that there are no black leaves by construction. Thus together with Lemma 43 the proof can be applied in this case.

Lemma 43. There can be no four-valent black vertices on the periphery of $H$.

Proof. Such a vertex could not be on $C_{i-1}$ as it would be four-valent there, contradicting Theorem 27, so it would have to be on $C_{i}$ or $C_{i+1}$. However, since there are no vertices in $H$ except on its periphery, this would contradict the minimal distance path assumption.

Thus there are two black two-valent vertices. We will show that one of these sits on $C_{i}$ and the other on $C_{i+1}$ in such a way that they are also two-valent there.

Suppose by way of contradiction one of these, say $v_{1}$, appears on $C_{i-1}$; let $u_{1}$ and $u_{2}$ be its two white neighbors there. Then there is a square face in $H$ containing $u_{1}, v_{1}, u_{2}$, and some black vertex $v_{2}$, which must lie on the periphery of $H$ because there are no interior vertices. However, this vertex cannot appear on $C_{i-1}$ as it would either be four-valent there or it would contradict the minimality assumption, and it cannot appear on $C_{i}$ or $C_{i+1}$ as it would contradict the minimality assumption there, as well.

Then the two-valent black vertex $v_{1}$ on the periphery of $H$ must appear on paths on either $C_{i}$ or $C_{i+1}$, say $C_{i}$. However, it cannot appear as an internal vertex on either of these paths, as there would be a square face in $H$ containing it together with its two white neighbors as well as some other black vertex $v_{2}$. This vertex cannot lie on $C_{i-1}$ or $C_{i}$ as it would contradict the minimality assumption, and it cannot lie on $C_{i+1}$ as it would contradict the assumption that $C_{i}$ and $C_{i+1}$ are not connected by an edge. 
Not only must $v_{1}$ appear as an endpoint of the path on $C_{i}$, but it must appear as the trivial path itself on $C_{i}$; otherwise there is a square face in $H$ containing it together with its white neighbors as well as some other black vertex $v_{2}$. This vertex cannot lie on $C_{i-1}$ as it would either contradict the minimality assumption or be four-valent on $C_{i-1}$, it cannot lie on $C_{i}$ as it would contradict the minimality assumption, and it cannot lie on $C_{i+1}$ as it would contradict the assumption that $C_{i}$ and $C_{i+1}$ are not connected by an edge.

Thus the path $C_{i}$ is in fact trivial, and $v_{1}$ is the only vertex from the periphery of $H$ that is on it. Since it is two-valent in $H$ and in the interior of the interior graph $\Gamma_{i-1}$, it must be four-valent in $\Gamma_{i-1}$ with its other two edges as edges of $C_{i}$. Thus it is two-valent in $\Gamma_{i}$.

The second two-valent black vertex on the periphery of $H$ can then only appear as a two-valent black vertex on $C_{i+1}$ following the procedure above, completing the proof.

Remark 44. Consider the number of faces in $H$. When $H$ is just a single face, the two black vertices on $C_{i}$ and $C_{i+1}$ can be thought of as handles. When $H$ contains more faces, these handles can be thought of as "stretched" like an accordion to enlarge the paths on $C_{i-1}$.

Similarly, such a region $H$ can be "compressed" like an accordion into a single face by a local move imitating the Simply Connected Region Reduction Proposition 38 without altering the structure of the cycles within $\Gamma_{i-1}$. This cannot be employed globally as it may effect $\Gamma_{i-2}$.

Remark 45. If $\Gamma_{i-1} \backslash C_{i-1}$ has more than two connected components, they must be arranged in a tree-like fashion and so the Accordion Proposition 42 can be applied to the pair of components along each edge of this tree.

There is one last way for several interior cycles to appear, and that is from breaking cutvertices. We show how new two-valent black vertices arise in this context.

Proposition 46. Party Hat Proposition. Suppose that when a white cutvertex $u_{1}$ is broken in the interior graph $\Gamma_{i-1}$ it results in two components, each containing cycles after pruning leaf edges and breaking additional cutvertices. Let $C_{i}$ and $C_{i+1}$ be the two cycles, one in each component with $C_{i}$ containing the original cutvertex, that are closest to each other using the usual notion of distance on the graph. Then this results in an extra pair of black two-valent vertices, both on $C_{i+1}$.

As in Figure 14, the interior cycle $C_{i}$ appears as the puff ball at the top of the so-called party hat worn by the interior cycle $C_{i+1}$.

Proof. First use the Leaf Edge Reduction Proposition 35 to remove the leaf edges. Observe that this does not change the structure of the cycles in consideration here.

Recall from Lemma 29 that after deleting the cutvertex $u_{1}$ exactly one of the components has an odd number of vertices; say this is cycle $C_{i}$. Look at the black neighbors of $u_{1}$ that are on $C_{i+1}$ and consider their cyclic order around $u_{1}$. 


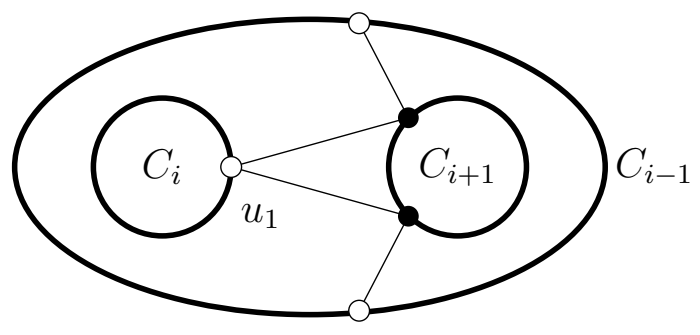

Figure 14: A party hat arises if a cutvertex is broken when $C_{i-1}$ is deleted from $\Gamma_{i-1}$.

Lemma 47. The cutvertex $u_{1}$ has valence at least two in the induced subgraph together with the cycle $C_{i+1}$.

Proof. Suppose by way of contradiction that $u_{1}$ has valence one in $C_{i+1} \cup\left\{u_{1}\right\}$; let $v_{1}$ be its neighbor on $C_{i+1}$. Since $v_{1}$ is four-valent in $\Gamma_{i-1}$, it has one extra neighbor besides $u_{1}$ and the two on $C_{i+1}$. If this is outside of $\Gamma_{i+1}$ consider the neighbor $u_{2}$ of $v_{1}$ on the opposite side of $u_{1}$; otherwise choose either neighbor.

Then the square face outside the interior graph $\Gamma_{i+1}$ containing $u_{1}, v_{1}$, and $u_{2}$ has one additional black vertex $v_{2}$. This black vertex cannot be on $C_{i-1}$ as it would be four-valent there; it cannot be on $C_{i}$ as it would give way to a larger cycle containing both $C_{i}$ and $C_{i+1}$. If it is on $C_{i+1}$, this contradicts the assumption that $u_{1}$ has valence one there.

There are no leaf edges to consider by the Leaf Edge Reduction Proposition 35, so $v_{2}$ must be on some other cycle $C_{i+2}$, where it is two-valent.

Then following the proof of Sublemma 32, this situation again forces two new black vertices that must be handled according to the above arguments. What is more is that no new black vertex created can appear on a previous interior cycle, as it would alter the cycle structure. Thus ultimately all new interior cycles and leaf edges are exhausted, and there are no remaining options for the black vertex, a contradiction.

By the lemma above, there are at least two black neighbors of $u_{1}$ on $C_{i+1}$, and so we may consider just the two on the outside (on either side) of this cyclic order; call these $v_{1}$ and $v_{2}$.

We show that $v_{1}$ and $v_{2}$ are two-valent in $C_{i+1}$ and thus there are no other two-valent black vertices on $C_{i+1}$ by the Periphery Proposition Lemma 31 .

If not $v_{1}$ must be three-valent following the lemma. Then there is a square face in $\Gamma_{i-1}$ outside of $\Gamma_{i}$ and $\Gamma_{i+1}$ containing $u_{1}, v_{1}$, and a white neighbor of $v_{1}$ on $C_{i+1}$ that also contains some black vertex $v_{3}$.

This black vertex $v_{3}$ cannot be on $C_{i-1}$ as it would be four-valent there; it cannot be on $C_{i}$ or $C_{i+1}$ as this would change the cycle structure. Since there are no leaf edges, it must be on some new $C_{i+2}$.

Then following the proof of Sublemma 32, this situation again forces two new black vertices that must be handled according to the above arguments. What is more is that no new black vertex created can appear on a previous interior cycle, as it would alter the 
cycle structure. Thus ultimately all new interior cycles and leaf edges are exhausted and there are no remaining options for the black vertex, a contradiction.

Remark 48. Note that the two new black two-valent vertices appear on the same component $C_{i+1}$. Since by the Periphery Proposition Lemma 31 there can only be two such vertices, no others are present.

The above results can be summarized by the following theorem.

Theorem 49. Suppose there is a black two-valent vertex on the interior cycle $C_{i}$ in the interior graph $\Gamma_{i-1}$. Then it resulted from excatly one of the following:

- a black two-valent vertex on $C_{i-1}$,

- an "accordion" together with a black two-valent vertex on some other $C_{i+1}$, or

- a "party hat" together with the other black two-valent vertex on $C_{i}$.

Proof. First use the Leaf Edge Reduction Proposition 35 to remove the leaf edges. Observe that this does not change the structure of the cycles in consideration here and in particular does not affect two-valent black vertices.

The Periphery Proposition 3 accounts for the two black two-valent vertices on $C_{1}$. The Stacking Proposition 40 accounts for the two black two-valent vertices on each subsequent $C_{i}$ unless there are several interior cycles within some $C_{i-1}$. These arise due to either disconnected components in $\Gamma_{i-1} \backslash C_{i-1}$ or breaking cutvertices. The Accordion Proposition 42 accounts for two additional black two-valent vertices for each additional component, and the Party Hat Proposition 46 accounts for two additional black two-valent vertices for each additional cycle after breaking cutvertices.

By the Periphery Proposition Lemma 31 there can be no more such black two-valent vertices.

Now that we have the appropriate notions of accordions and party hats, the following reduction propositions can be introduced.

Proposition 50. Accordion Reduction Proposition. Suppose that an accordion arises when $C_{i-1}$ is deleted from the interior graph $\Gamma_{i-1}$ as in the Accordion Proposition 42. Furthermore, suppose that the interior cycle $C_{i+1}$ is connected to $C_{i}$ by the accordion but is not connected to any other cycles. Then the interior graph $\Gamma_{i-1}$ can be reduced to one without $C_{i+1}$ by a local move as depicted in Figure 15.

Proof. First use the Leaf Edge Reduction Proposition 35 to remove the leaf edges. Observe that this does not change the structure of the cycles in consideration here.

Recall that the accordion and the cycle $C_{i+1}$ share one black vertex $v_{1}$ that is two-valent on $C_{i+1}$. Then since there are two such vertices on each cycle by the Periphery Proposition Lemma 31, there is some other black two-valent vertex $v_{2}$ on $C_{i+1}$. By Theorem 49 this must arise from a black two-valent vertex $v_{3}$ on $C_{i-1}$ since $C_{i+1}$ is not connected to any other cycles. 


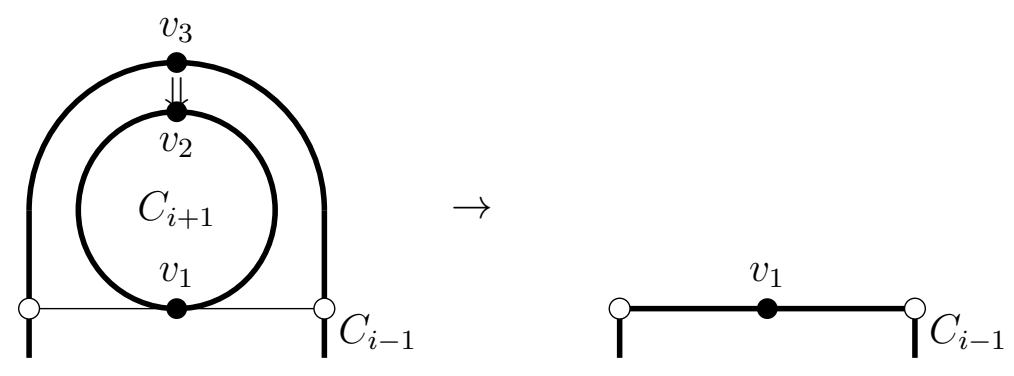

Figure 15: Applying the Accordion Reduction Proposition 50.

Delete all faces on and inside $C_{i+1}$ except the one that is part of the accordion on black vertex $v_{1}$. Then this black vertex is now two-valent on $C_{i-1}$, replacing the former one $v_{3}$. Furthermore, the cycle structure in $\Gamma_{i-1}$ outside of $C_{i+1}$ remains the same.

Remark 51. One cannot employ the Accordion Reduction Proposition 50 while considering the larger interior graph $\Gamma_{i-2}$. Moreover, employing this reduction move ignores all of $\Gamma_{i+1}$.

Proposition 52. Party Hat Reduction Proposition. Suppose that a party hat arises when $C_{i-1}$ is deleted from the interior graph $\Gamma_{i-1}$ as in the Party Hat Proposition 46. Furthermore, suppose that the interior cycle $C_{i+1}$ is connected to $C_{i}$ by the party hat but is not connected to any other cycles. Then the interior graph $\Gamma_{i-1}$ can be reduced to one without $C_{i+1}$ by a local move as depicted in Figure 16.

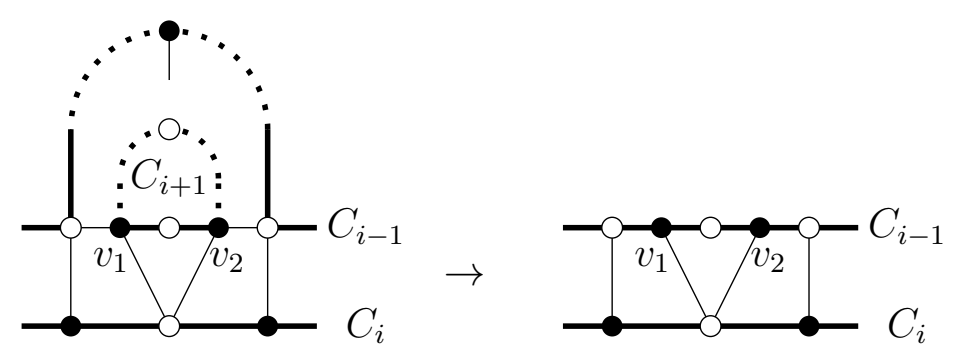

Figure 16: Applying the Party Hat Reduction Proposition 52.

Proof. First use the Leaf Edge Reduction Proposition 35 to remove the leaf edges. Observe that this does not change the structure of the cycles in consideration here.

Recall that the party hat and the cycle $C_{i+1}$ share two black vertices $v_{1}$ and $v_{2}$ that are two-valent on $C_{i+1}$. Then since there are only two such vertices on each cycle by the Periphery Proposition Lemma 31, there are no others. Thus by the Stacking Proposition 40 there cannot be any two-valent black vertices on $C_{i-1}$ in the vicinity of $C_{i+1}$.

Delete all faces on and inside $C_{i+1}$ except for those that are part of the cone of the party hat along with the two that are adjacent to it (one on $v_{1}$ and one on $v_{2}$ ). Then 
these black vertices $v_{1}$ and $v_{2}$ are now on $C_{i-1}$ and three-valent there. Furthermore, the cycle structure and black vertices in $\Gamma_{i-1}$ outside of $C_{i+1}$ remain the same.

Remark 53. One cannot employ the Party Hat Reduction Proposition 52 while considering the larger interior graph $\Gamma_{i-2}$. Moreover, employing this reduction move ignores all of $\Gamma_{i+1}$.

These last two reduction moves will be used in the proof of Theorem 56 .

\section{The graph of perfect matchings via cycles}

These cycles $C_{i}$ emerge when the symmetric difference is taken of $\widehat{0}$ and $\widehat{1}$, the unique minimum and maximum elements in the graph $\mathcal{G}$ of perfect matchings of $\Gamma$ when seen as a lattice.

Decompose each cycle $C_{i}$ into two perfect matchings on the cycle subgraph: the collection $\mu_{i}^{0}$ of edges that traverse from black to white in a clockwise direction and the collection $\mu_{i}^{1}$ of edges that traverse from black to white in a counterclockwise direction. This orientation of course assumes the topological properties of the plane embedding of the original knot diagram.

Definition 54. A cycle is said to be $\left(\mu_{1}, \mu_{2}\right)$-alternating if the edges appear alternately in the two matchings $\mu_{1}$ and $\mu_{2}$.

Theorem 55. Each $C_{i}$ is $(\widehat{0}, \widehat{1})$-alternating. Furthermore, the set of leaf edges is exactly the set of edges that appear in both of these states.

Proof. Consider first the union of the $\mu_{i}^{0}$ together with the leaf edges; to see this is the unique least element $\widehat{0}$ of Kauffman's clock lattice $\mathcal{L}$, it is enough to show that it cannot be counterclocked. A counterclock move can only occur when two edges $e_{i}$ and $e_{j}$ (going clockwise from white vertex to black vertex on the boundary of the same square face $f$ ) belong to the pefect matching.

Recall that for an edge $e_{i}$ in $\widehat{0}$ to belong to a cycle $C_{i}$, it must go from black vertex to white vertex along the face within $\Gamma_{i}$. Thus if the edge $e_{i}$ belongs to $C_{i}$, the face $f$ must lie outside of $\Gamma_{i}$; if this is the case for both $e_{i}$ and $e_{j}$, the cycles $C_{i}$ and $C_{j}$ are neighboring, but then the cycles $C_{i}$ and $C_{j}$ could have been extended through this face $f$ creating a larger cycle bounding $\Gamma_{i} \cup \Gamma_{j} \cup\{f\}$, a contradiction. Then $e_{j}$ must be a leaf edge; if $e_{i}$ is not, the edge $e_{j}$ lies outside of $C_{i}$, but this could have been extended to a larger cycle bounding $\Gamma_{i} \cup\{f\}$ by the Inchworm Lemma 30. Then $e_{i}$ is a leaf edge, as well, and a new cycle $C_{i j}^{\prime}$ could have been created with interior $\Gamma_{i j}^{\prime}=f$, a contradiction.

The proof that the union of the $\mu_{i}^{1}$ together with the leaf edges cannot be clocked is similar.

See for example the clocked and counterclocked states of a knot diagram for $K 11 n 157$ in Figure 17. Notice that in particular when these are overlapped there are two concentric cycles and a single leaf edge in the center, as in Figure 7 above.

Now we arrive at the main theorem. 

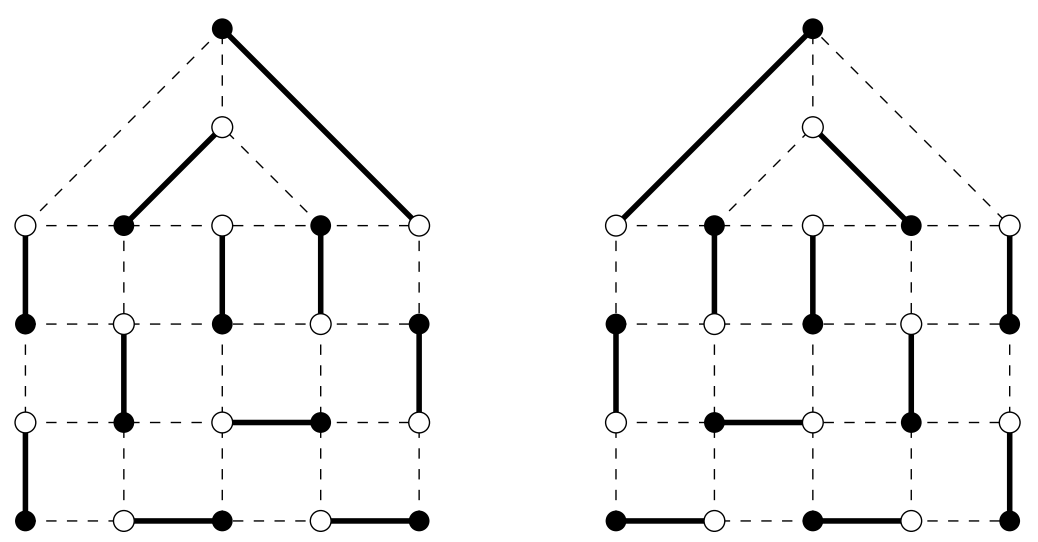

Figure 17: The clocked and counterclocked states of a diagram for $K 11 n 157$.

Theorem 56. Consider the balanced overlaid Tait graph $\Gamma$ obtained from a knot diagram, and let $s\left(C_{i}\right)$ be the number of square faces within the interior graph $\Gamma_{i}$. Then

$$
\sum_{i} s\left(C_{i}\right)=h
$$

gives the height of the clock lattice.

In particular, $s\left(C_{1}\right)$ is equal to the original number of square faces of the graph $\Gamma$.

For the example appearing in Figures 7 and 17 for a diagram of the knot $K 11 n 157$, there are fifteen square faces in the original graph $\Gamma=\Gamma_{1}$ and five square faces in the interior graph $\Gamma_{2}$, and so and the height of the clock lattice is twenty.

Proof. Since $\mathcal{G}$ is connected, there is always at least one possible flip move to make, so $s\left(C_{i}\right) \neq 0$.

Proceed by induction on $k$, the number of cycles. The base case is handled by Lemma 57, which also shows that the Simply Connected Region Reduction Proposition 38 does not affect Equation 1 in Lemma 58. The induction hypothesis on an annulus is then shown in Lemma 59, barring the Accordion Reduction Proposition 50 handled in Lemma 60, the Party Hat Reduction Proposition 52 handled in Lemma 61, and the Leaf Edge Reduction Proposition 35 handled in Lemma 62.

Lemma 57. Base Case: One Cycle. A knot diagram with exactly one connected cycle $C$ has clock lattice height of $s(C)$.

Proof. Induct on the number of squares $s(C)$ within the only cycle $C$; as a base case, a single square has height one.

Suppose there are $s(C)=m$ squares within the cycle and that the induction hypothesis holds for all cycles containing fewer than $m$ squares. Choose some square face $f_{1}$ sharing at least one edge with $C$; this produces a new cycle $C^{\prime}=C \triangle f_{1}$ within $C$ by the symmetric difference. 


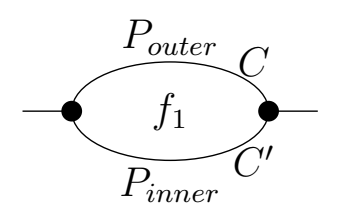

(B)

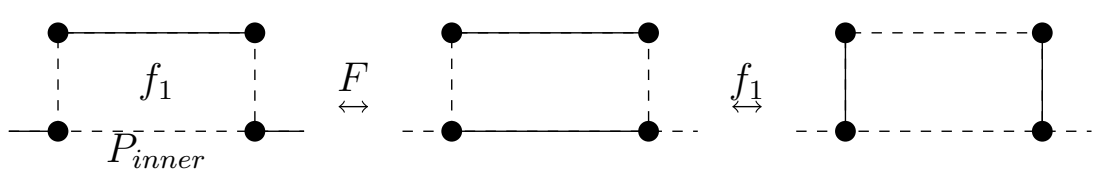

$(C)$

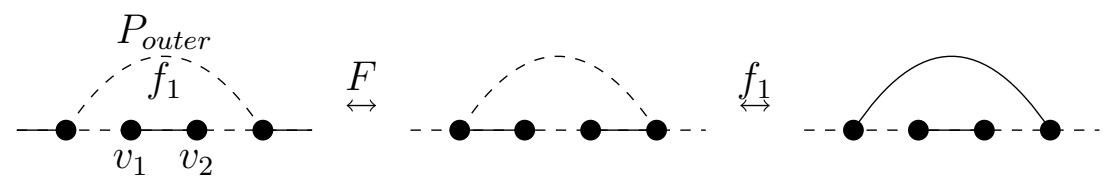

$(D)$

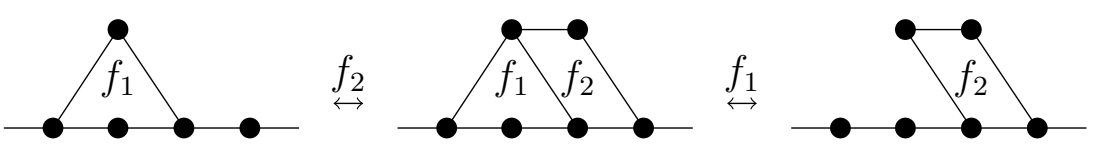

Figure 18: Lemma 57 provides the base case for Theorem 56 , adding new square face $f_{1}$. The vertex sets are undistinguished.

The square face $f_{1}$ cannot share all its four edges with $C$. It must share consecutive edges; otherwise $C^{\prime}$ would not be connected and there would be more than one cycle.

Let $P_{\text {outer }}$ (respectively $P_{\text {inner }}$ ) be the path formed by those consecutive edges on $f_{1}$ shared with $C$ (respectively $C^{\prime}$ ), and partition this path into $\mu_{\text {outer }}^{0}$ and $\mu_{\text {outer }}^{1}$ (respectively $\mu_{\text {inner }}^{0}$ and $\mu_{\text {inner }}^{1}$ ), those edges that traverse from white to black in a clockwise direction around $C$ (respectively $C^{\prime}$ ) and, respectively, those edges that traverse from white to black in a counterclockwise direction. Observe that $P_{\text {outer }}$ is "outside" of $f_{1}$ and $P_{\text {inner }}$ is on the "inside", as in Figure 18 (A), where black and white vertices are undistinguished throughout. By the induction hypothesis, there must be some sequence $F$ of $m-1$ flip moves that transfer $\mu_{C^{\prime}}^{0}$ to $\mu_{C^{\prime}}^{1}$ (and in particular $\mu_{\text {inner }}^{0}$ to $\mu_{\text {inner }}^{1}$ ), as $P_{\text {inner }}$ belongs to $C^{\prime}$.

First suppose that $\left|P_{\text {inner }}\right|=1$; then either $f_{1}$ followed by $F$ or $F$ followed by $f_{1}$ transfers $\mu_{C}^{0}$ to $\mu_{C}^{1}$ in a total of $m$ moves, as in Figure 18 (B). The other cases involve the inclusion of new leaf edges.

Supposing that $\left|P_{\text {inner }}\right|=3$, there are two additional vertices $v_{1}$ and $v_{2}$ in $P_{\text {inner }}$ that are inside of $C$ but not contained in $C$. If there are no other leaf edges inside of $C$, then the clocked state of $C$ is $\mu_{C}^{0}$ together with the leaf edge $\ell$ between $v_{1}$ and $v_{2}$. Then as before either $f_{1}$ followed by $F$ or $F$ followed by $f_{1}$ transfers $\mu_{C}^{0} \amalg \ell$ to $\mu_{C}^{1} \amalg \ell$ in $m$ moves, as in Figure $18(\mathrm{C})$. Otherwise it may be possible for this leaf edge to share a face with two other vertices in a leaf edge; however this would form a new cycle $C^{\prime \prime}$ within $C$, a contradiction, and thus there is just a single additional leaf edge.

Removing a square $f_{1}$ with $\left|P_{\text {inner }}\right|=2$ yields a bipartite graph with unequally-sized vertex sets; thus two such squares $f_{1}$ and $f_{2}$ need to be removed at a time since no other case allows for unequally-sized vertex sets. These squares may not share more than two 
of the same edges since the "inside" paths $P_{\text {inner }}$ cannot overlap, and the case where both $P_{\text {inner }}$ are the same two edges violates Theorem 27 . These squares $f_{1}$ and $f_{2}$ cannot share two of the same edges, as this would not yield equally-sized vertex sets. When $f_{1}$ and $f_{2}$ are disjoint this also violates Theorem 27.

Therefore $f_{1}$ and $f_{2}$ must share a single edge as depicted in Figure 18 (D); deleting first $f_{2}$ (as on the left side) and then $f_{1}$ yields the same result as deleting first $f_{1}$ (as on the right side), which has $\left|P_{\text {inner }}\right|=3$, and then $f_{2}$, which has $\left|P_{\text {inner }}\right|=1$; thus here the $\left|P_{i}\right|=2$ squares are unneccessary.

This can also be used to handle any simply connected region.

Lemma 58. Invariance of equality under the Simply Connected Region Reduction Proposition. The Simply Connected Region Reduction Proposition 38 does not affect the equality of Theorem 56.

Proof. Following the proof of Lemma 57 above for the $\left|P_{\text {inner }}\right|=1$ case, extend the single square $f_{1}$ to any set of simply connected squares. Extend the path $P_{\text {outer }}$ from length three in the case above to any odd length (since the graph is bipartite) in the natural way, and the result holds by applying Lemma 57 .

We proceed to the induction step: a single annulus between two cycles $C_{i-1}$ and $C_{i}$ with no leaf edges.

Lemma 59. Induction Hypothesis: Flipping an Annulus. Suppose the interior graph $\Gamma_{i-1}$ has exactly one cycle $C_{i}$ at the next level inside of it. Flipping all the square faces in $\Gamma_{i-1} \backslash \Gamma_{i}$ exactly once takes the local perfect matchings of $\mu_{i-1}^{0}$ and $\mu_{i}^{1}$ to those of $\mu_{i-1}^{1}$ and $\mu_{i}^{0}$.

Proof. Assume for now that there are no leaf edges in the annulus. These will be taken care of by another lemma below.

This annulus has outside face $C_{i-1}$ and inside face $C_{i}$. Because there are no leaf edges, all vertices are on $C_{i-1}$ and $C_{i}$, so this gives five types of square faces as in Figure 19. First observe that any edge between two vertices of $C_{i-1}$ (respectively two vertices of $C_{i}$ ) encloses a simply connected region of squares with $C_{i-1}$ (respectively $C_{i}$ ), and so by Lemma 58 this does not affect the equality of Theorem 56. This leaves only cases III, IV, and $\mathrm{V}$, where edges must be on $C_{i-1}$ or $C_{i}$, or must traverse them.

Partition these latter edges into two sets: those with a black vertex on $C_{i}$ and those with a black vertex on $C_{i-1}$. Consider the oriented dual edges to these edges, directed to the right when leaving the black vertex. Then every vertex in this "dual-in-the-annulus" graph has valence two, allowing for sources and sinks.

Begin by performing clock moves on all sources, following these oriented edges in the dual graph outwards, and performing a clock move on a sink when it is reached from both sides simultaneously. Thus each square is counted exactly once, and so this changes $\mu_{i-1}^{0}$ to $\mu_{i-1}^{1}$ and $\mu_{i}^{1}$ to $\mu_{i}^{0}$. 


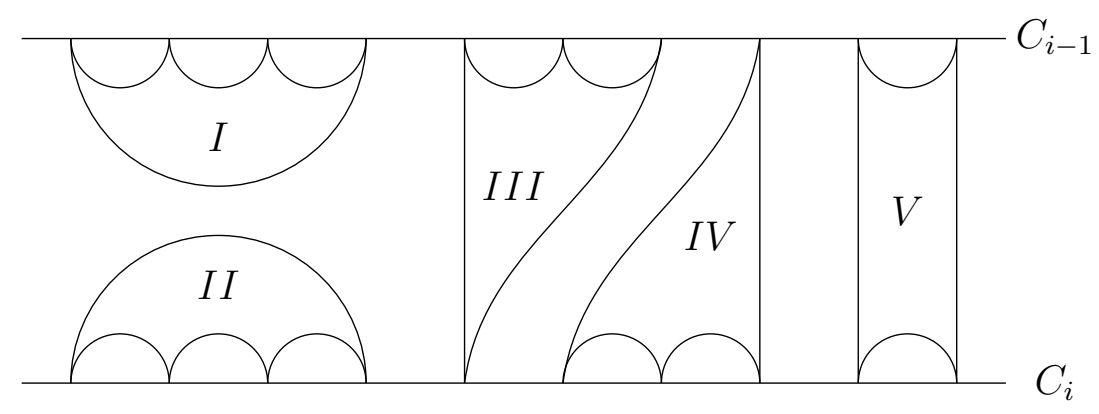

Figure 19: The five types of squares on an annulus with no leaf edges.

However it may be the case that there are several interior cycles at the same level; these occur with accordions and party hats. In each of these configurations, we consider a single additional cycle, turn it into an annulus, and then apply Lemma 59.

Lemma 60. Invariance of equality under the Accordion Reduction Proposition. The Accordion Reduction Proposition 50 does not affect the equality of Theorem 56 .

Proof. Consider Figure 15, and let $H$ be the induced subgraph containing $\Gamma_{i+1}$ and the path on the cycle $C_{i-1}$ that is deleted. This is almost an annulus; to turn this into an annulus $H^{\prime}$ one need only add a single square face $f$ at the two-valent vertex on the cycle $C_{i+1}$ that is on the periphery of $H$ as in Figure 20 .

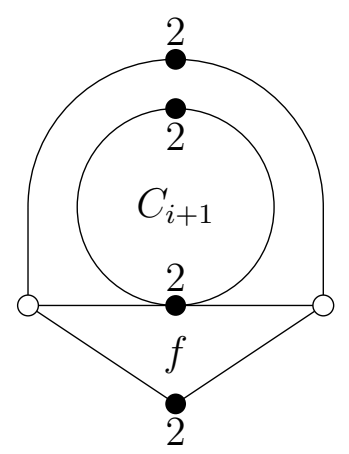

Figure 20: Adding a square face $f$ to the deleted subgraph $H$ of the Accordion Reduction Proposition 50 yields an annulus $H^{\prime}$.

Observe that $H^{\prime}$ has two black two-valent vertices by construction and since the remaining black vertices on the periphery were also on the periphery of $\Gamma_{i-1}$, this annulus $H^{\prime}$ satisfies the Periphery Proposition Lemma 31.

Thus we can apply Lemma 59 to show that each of the square faces in this annulus contribute exactly once to the height. It is easy to see that deleting the additional face $f$ does not affect this equality. 
Lemma 61. Invariance of equality under the Party Hat Reduction Proposition. The Party Hat Reduction Proposition 52 does not affect the equality of Theorem 56 .

Proof. As in the proof of Lemma 60, consider Figure 16, and let $H$ be the induced subgraph containing $\Gamma_{i+1}$ and the path on the cycle $C_{i-1}$ that is deleted. This again is almost an annulus; to turn this into an annulus $H^{\prime}$ one need only add some square faces $f_{1}, \ldots, f_{n}$ at the two black two-valent vertices on the cycle $C_{i+1}$ that is on the periphery of $H$ as in Figure 21.

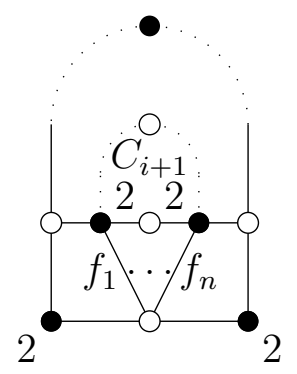

Figure 21: Adding square faces $f_{1}, \ldots, f_{n}$ to the deleted subgraph $H$ of the Party Hat Reduction Proposition 52 yields an annulus $H^{\prime}$.

Observe again that $H^{\prime}$ has two black two-valent vertices by construction and since the remaining black vertices on the periphery were also on $C_{i-1}$ or $C_{i+1}$, this annulus $H^{\prime}$ satisfies the Periphery Proposition Lemma 31.

Thus as above we can apply Lemma 59 to show that each of the square faces in this annulus contribute exactly once to the height. It is easy to see that deleting the additional faces $f_{1}, \ldots, f_{n}$ does not affect this equality.

Lastly we show that we can remove leaf edges to arrive at any of the cases above.

Lemma 62. Invariance of equality under the Leaf Edge Reduction Proposition. Equation 1 is preserved by the Leaf Edge Reduction Proposition 35.

Proof. Suppose there is a leaf edge in $\Gamma_{i-1}$ as in Figure 22, with square faces labelled $f_{1}$ to $f_{n}$ along the upper part of the figure leaving the two faces $f_{n+1}$ and $f_{n+2}$ on the lower part. Label the boundary $\partial H$ of this induced subgraph $H$ in a counterclockwise manner $e_{1} \cup P_{\text {upper }} \cup e_{n} \cup P_{\text {lower }}$ starting at the right. We suppose that faces outisde of the induced subgraph $H$ act as we need so that the edges in the figure can be considered in perfect matchings.

We first show that each square face in the induced subgraph $H$ must be flipped exactly once to transfer the edges from $\mu_{\partial H}^{0}$ to $\mu_{\partial H}^{1}$ where $\widehat{0} \leqslant \mu_{\partial H}^{0}<\mu_{\partial H}^{1} \leqslant \widehat{1}$ with partial order given by Kauffman's lattice.

Observe that the leaf edge belongs to the clocked state $\widehat{0}$ (and so no other edges on these vertices can belong to $\widehat{0}$ ); thus the only possible flip move here is $f_{1}$, since we may 
assume that $e_{1}$ is in $\mu_{\partial H}^{0}$. Then the faces $f_{2}, \ldots, f_{n-1}$ can be flipped successively along the upper part and the faces $f_{n+1}$ and $f_{n+2}$ can be flipped successively along the lower part. Finally $f_{n}$ can be flipped, and the matching on the boundary is indeed $\mu_{\partial H}^{1}$.

Next see that after the reduction move, each square in the new induced subgraph $H^{\prime}$ must be flipped exactly once, $f_{1}, \ldots, f_{n}$ successively, to transfer the edges from $\mu_{\partial H^{\prime}}^{0}$ to $\mu_{\partial H^{\prime}}^{1}$

Lastly observe that all of these square faces belong to the same interior graph $\Gamma_{i-1}$ but not to any smaller nested interior graphs $\Gamma_{i}, \Gamma_{i+1}, \ldots$ so they are counted exactly once per cycle.

If there are several leaf edges, one of these reduction moves may be performed at a time.

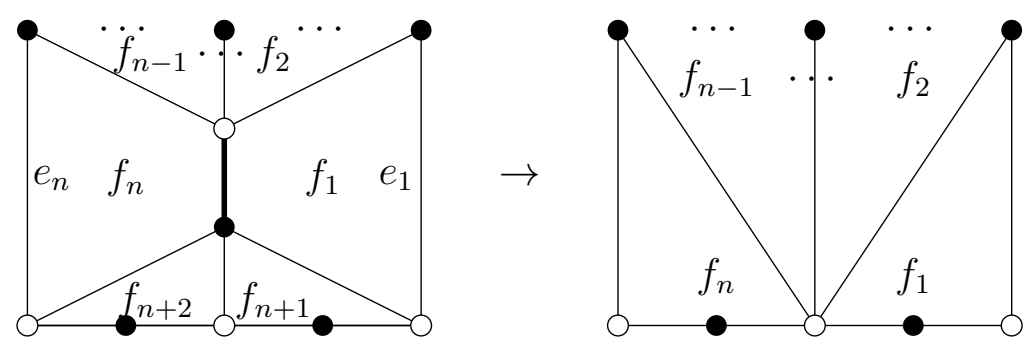

Figure 22: The Leaf Edge Reduction Proposition 35 already has a simply connected region $H$.

This completes the proof of the Main Theorem 56 .

\section{Examples}

Example 63. Abe (in [1]) considers a six crossing knot universe as a running example, displaying its clock lattice (with one edge missing) in Figure 7 . We translate this example to the graph of perfect matchings in Figure 23 below.

Example 64. Split links and other non-prime-like diagrams. Given such a diagram, one can use a trick similar to one in [2], pulling some strand from one side over or under a strand on the other side. Repeat several times if necessary to produce a prime-like diagram like those considered above.

Example 65. The number of cycles is not a knot invariant. Consider for example the two projections of the knot $10_{44}$ found in Knot Info [13] and the Knot Atlas [7]. Taking "similar" starred regions, the balanced overlaid Tait graph of the first has two cycles but that of the second has only one. The graph of perfect matchings of the first has height $11+1=12$ and that of the second has height 9 .

However, the number of cycles may be used to obtain an upper bound for the bridge number of the knot if the following conjecture is true. 


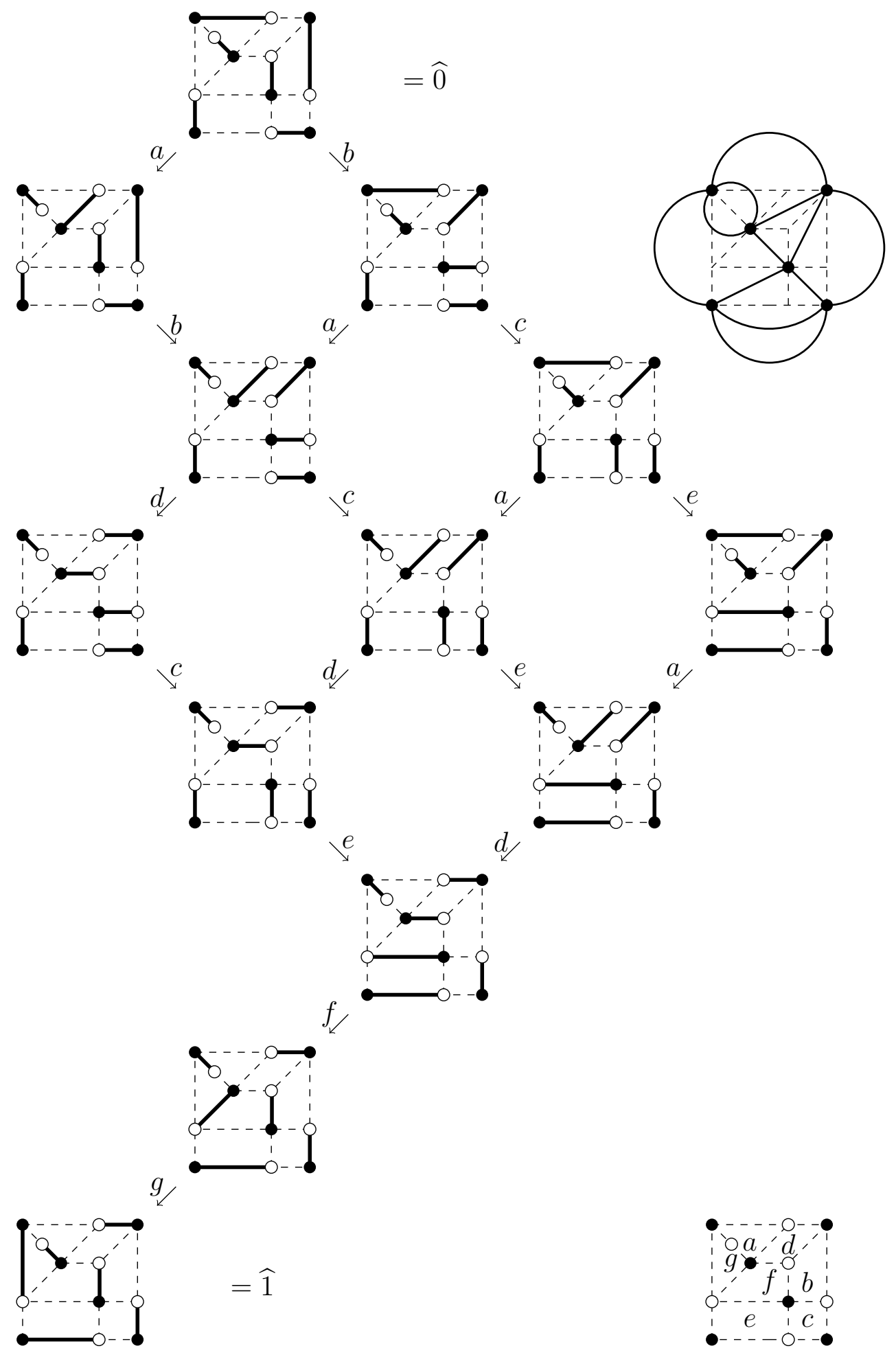

Figure 23: Translating an example from Abe. 
Conjecture 66. The number of cycles is related to the bridge number of the universe.

This is supported by notions in the next subsection.

\subsection{Application to grid graphs and harmonic knots}

Let $\Gamma_{m, n}$ be the grid graph of $m \times n$ squares with $m, n$ both odd. This ensures that the graph has equal-sized vertex sets. Let us say that $m \leqslant n$, so that $\min =\min \{m, n\}=m$. Then $(1 / 2)(\min -1)=\frac{m-1}{2},(1 / 2)(\min -1)+1=\frac{m+1}{2}$, and $2(1 / 2)(\min -1)+1=m$.

Corollary 67. The height of the clock lattice for the square grid graph $\Gamma_{m, m}$ is the $m$-th tetrahedral number $(1 / 6) m(m+1)(m+2)$.

Proof. Let $\min =\min \{m, n\}$. By Theorem 56 above, the height of the clock lattice is:

$$
\begin{aligned}
& =\sum_{i=0}^{(1 / 2)(\min -1)}(m-2 i)(n-2 i)=\sum_{i=0}^{(1 / 2)(\min -1)}\left[m n-2 i(m+n)+4 i^{2}\right] \\
& =\frac{m n(\min +1)}{2}-\frac{(m+n)(\min -1)(\min +1)}{4}+\frac{\min (\min -1)(\min +1)}{6} \\
& =\frac{\left(3 m^{2} n+6 m n-m^{3}+m+3 n\right)}{12} \text { setting } \min =m \\
& =\frac{(m+1)[3 n(m+1)-m(m-1)]}{12} \\
& =\frac{(m)(m+1)(m+2)}{6} \text { in the case where } n=m .
\end{aligned}
$$

Observe that this is the $m$-th tetrahedral number, which is [A000292] on the On-Line Encyclopedia of Integer Sequences [19].

A harmonic curve is one that admits a parametrization whose three coordinate functions $x=x_{1}, y=x_{2}, z=x_{3}$ are the classical Chebyshev polynomials $T_{x_{i}}(t)$ defined by $T_{n}(\cos t)=\cos (n t)$. A Chebyshev curve is one whose third coordinate function $T_{x_{3}}(t+\varphi)$ has a phase shift. Identifying the ends of a non-singular harmonic curve, one obtains a harmonic knot $H\left(x_{1}, x_{2}, x_{3}\right)$ if and only if the three parameters $x_{i}$ are pairwise coprime integers (Comstock 1897 [18] or see also [41] or [25]).

Koseleff and Pecker found in [39] that the trefoil could be parametrized in such a way, leading them to study harmonic knots in [40, 41, 42]. Harmonic knots are polynomial analogues of the famous Lissajous knots studied in $[10,11,20,31,34,45,46]$; however, the figure-eight knot is not a Lissajous knot but is the harmonic knot $H(3,5,7)$.

The interested reader may find many examples of Chebyshev knot diagrams on the website of Vincent-Pierre Koseleff:

http://www.math.jussieu.fr/ ${ }^{\sim}$ koseleff/knots/kindex1.html

Theorem 68. [41, Theorem 3] Every knot has a projection which is a Chebyshev plane curve. 
This is a consequence of another result in their paper involving bridge number, further confirming the suspicions of Conjecture 66.

Remark 69. The harmonic knot $H\left(x_{1}, x_{2}, x_{3}\right)$ has a balanced overlaid Tait graph $\Gamma$ that is the $\left(x_{1}-2\right) \times\left(x_{2}-2\right)$ grid graph $\Gamma_{x_{1}-2, x_{2}-2}$. Thus every knot has a projection whose balanced overlaid Tait graph $\Gamma$ is a grid graph!

We conclude with one other application.

\subsection{Relationship with discrete Morse theory}

This subject was introduced in 1995 by Forman (see for example [24]) to apply the power of the classical version to combinatorially defined complexes. The critical points found in the smooth version can be determined combinatorially by collapsing pairs of $i$ - and $i+1$-dimensional cells in the complex $C$, and these collapses are described (and ordered) using the following map.

A discrete Morse function is a weakly increasing map $f:(C, \subseteq) \rightarrow(\mathbb{Z}, \leqslant)$ such that $\left|f^{-1}(n)\right| \leqslant 2$ for all $n \in \mathbb{Z}$ and such that $f(\sigma)=f(\tau)$ implies that one of $\sigma, \tau$ is a face of the other. A critical cell for a discrete Morse function $f$ is face of $C$ at which $f$ is injective.

This subject has been studied in many contexts, including by Chari in [15] for shellability, by Babson and Hersh in [5] for lexicographic orders, by Kozlov in [43] for free chain complexes, by Welker in [56] for free resolutions, by Engström in [23] for Fourier transforms, by Ayala, Fernández, Fernández-Ternero, and Vilches in [4] for graphs, by Benedetti in [9] for homology, and by Salvetti, Gaiffi, and Mori in [26, 27] for line arrangements and in [50] for configuration spaces. It was recently the topic of a summer school at the Institut Mittag-Leffler outside of Stockholm, Sweden organized by Benedetti and Engström.

Consider a 2-complex $\Delta$ of the 2 -sphere, not necessarily simplicial, as we allow multiple 1-faces with the same two endpoints, 1-faces with the same single endpoint, and 2-faces of length less than three. This can be realized as a(n unsigned) plane graph $G$. Associate to this a knot universe $U$ described above. Then the face poset $\mathcal{F}(\Delta)$ of the complex can be realized as $\widehat{\Gamma}$. Following discrete Morse theory, a(n elementary) collapse on $\Delta$ can be realized as an edge in $\widehat{\Gamma}$. The deletion of the two starred regions in $\widehat{\Gamma}$ to obtain $\Gamma$ corresponds with choosing the two critical cells, one of dimension two and the other of dimension one.

Then a shelling of $\Delta$ with chosen critical cells corresponds with a discrete Morse function $f$ on $\Delta$ with these chosen critical cells, which corresponds with a perfect matching of $\Gamma$, which corresponds with two rooted spanning trees $T$ of $G$ and $T^{C}$ of the dual graph $G^{*}$, which correspond with a series of collapses together with a series of endocollapses (collapsing in the dual).

Proposition 70. Perfect matchings on $\Gamma$ constructed from a knot diagram $D$ correspond to discrete Morse functions on the 2-complex $\Delta$ of the 2-sphere. 
Proof. This follows by construction with the Tait graph $G$ coming from the 1-skeleton of $\Delta$ and with $\widehat{\Gamma}$ as the face poset $\mathcal{F}(\Delta)$ of the complex.

\section{Acknowledgements}

Inspiration for this project arose from three places: the Combinatorics group at BarIlan University and specifically a seminar talk by Roy Ben-Ari on part of his Masters of Science thesis [8] under the supervision of Ron Adin and Yuval Roichman; a preprint [1] by Yukiko Abe of Tokyo Institute of Technology containing some results of her Masters thesis; and the first author's graduate work $[16,17]$ at Louisiana State University on the balanced overlaid Tait graph together with his familiarity with Kauffman's clock lattice. The first author would also like to thank LSU VIGRE for sponsoring the first Baton Rouge Young Topologists Research Retreat in January 2012 whose central theme was the graph $\Gamma$ discussed below, Kate Kearney with whom he co-organized the workshop, and Cody Armond who contributed to many helpful conversations throughout.

\section{References}

[1] Yukiko Abe. The clock number of a knot. arXiv:1103.0072, 2011.

[2] Susan Abernathy, Cody Armond, Moshe Cohen, Oliver T. Dasbach, Hannah Manuel, Chris Penn, Heather M. Russell, and Neal W. Stoltzfus. A reduced set of moves on one-vertex ribbon graphs coming from links, Proc. Am. Math. Soc., 142(3):737-752, 2014.

[3] Christos A. Athanasiadis and Yuval Roichman. The absolute order of a permutation representation of a Coxeter group. J. Algebr. Comb., 39(1):75-98, 2014.

[4] R. Ayala, L. M. Fernández, D. Fernández-Ternero, and J. A. Vilches. Discrete Morse theory on graphs. Topology Appl., 156(18):3091-3100, 2009.

[5] Eric Babson and Patricia Hersh. Discrete Morse functions from lexicographic orders. Trans. Amer. Math. Soc., 357(2):509-534, 2005.

[6] John A. Baldwin and Adam Simon Levine. A combinatorial spanning tree model for knot Floer homology. Adv. Math., 231(3-4):1886-1939, 2012.

[7] Dror Bar-Natan, Scott Morrison, and et al. The Knot Atlas. http://katlas.org.

[8] Roy Ben-Ari. Combinatorial parameters on matchings in complete graphs. M. Sc. Thesis, Bar-Ilan University, 2010.

[9] Bruno Benedetti. Discrete Morse theory is at least as perfect as Morse theory. arXiv:1010.0548v4, 2010.

[10] M. G. V. Bogle, J. E. Hearst, V. F. R. Jones, and L. Stoilov. Lissajous knots. J. Knot Theory Ramifications, 3(2):121-140, 1994.

[11] Adam Boocher, Jay Daigle, Jim Hoste, and Wenjing Zheng. Sampling Lissajous and Fourier knots. Experiment. Math., 18(4):481-497, 2009. 
[12] Robert Burton and Robin Pemantle. Local characteristics, entropy and limit theorems for spanning trees and domino tilings via transfer-impedances. Ann. Probab., 21(3):1329-1371, 1993.

[13] J. C. Cha and C. Livingston. KnotInfo: Table of Knot Invariants. http://www. indiana.edu/ knotinfo.

[14] Abhijit Champanerkar and Ilya Kofman. Spanning trees and Khovanov homology. Proc. Amer. Math. Soc., 137(6):2157-2167, 2009.

[15] Manoj K. Chari. On discrete Morse functions and combinatorial decompositions. Discrete Math., 217(1-3):101-113, 2000.

[16] Moshe Cohen. A determinant formula for the Jones polynomial of pretzel knots. J. Knot Theory Ramifications, 21(6):23pp., 2012.

[17] Moshe Cohen, Oliver T. Dasbach, and Heather M. Russell. A twisted dimer model for knots. Fundam. Math., 225(1):57-74, 2014.

[18] Elting H. Comstock. The real singularities of harmonic curves of three frequencies. Transactions of the Wisconsin Academy of Sciences, Arts and Letters XI, 452-464, 1896-1897.

[19] Contributors. The On-Line Encyclopedia of Integer Sequences. http://oeis.org, Sequence A000292.

[20] Peter R. Cromwell. Knots and links. Cambridge University Press, Cambridge, 2004.

[21] Oliver T. Dasbach, David Futer, Efstratia Kalfagianni, Xiao-Song Lin, and Neal W. Stoltzfus. The Jones polynomial and graphs on surfaces. J. Combin. Theory Ser. B, 98(2):384-399, 2008.

[22] Reinhard Diestel. Graph theory, third ed., Graduate Texts in Mathematics, 173. Springer-Verlag, Berlin, 2005.

[23] Alexander Engström. Discrete Morse functions from Fourier transforms. Experiment. Math., 18(1):45-53, 2009.

[24] Robin Forman. A user's guide to discrete Morse theory. Sém. Lothar. Combin. 48:35pp., 2002

[25] Gene Freudenburg and Jenna Freudenburg. Curves defined by Chebyshev polynomials. arXiv:0902.3440v1, 2009.

[26] Giovanni Gaiffi, F. Mori, and Mario Salvetti. Minimal CW-complexes for complements to line arrangements via discrete Morse theory. Topology of algebraic varieties and singularities, Contemp. Math., 538:293-308. Amer. Math. Soc., Providence, RI, 2011.

[27] Giovanni Gaiffi and Mario Salvetti. The Morse complex of a line arrangement. J. Algebra, 321(1):316-337, 2009.

[28] Patrick M. Gilmer and Richard A. Litherland. The duality conjecture in Formal Knot Theory. Osaka J. Math., 23:229-247, 1986. 
[29] Joshua Greene. A spanning tree model for the Heegaard Floer homology of a branched double-cover. J. Topol., 6(2):525-567, 2013.

[30] C. Hernando, F. Hurtado, and Marc Noy. Graphs of non-crossing perfect matchings. Graphs Combin., 18(3):517-532, 2002.

[31] Jim Hoste and Laura Zirbel. Lissajous knots and knots with Lissajous projections. Kobe J. Math., 24(2):87-106, 2007.

[32] Stephen Huggett, Iain Moffatt, and Natalia Kaur Virdee. On the Seifert graphs of a link diagram and its parallels. Math. Proc. Camb. Philos. Soc., 153(1):123-145, 2012.

[33] Thomas C. Jaeger. A remark on Roberts' totally twisted Khovanov homology. J. Knot Theory Ramifications, 22(6), 2013.

[34] Vaughan F. R. Jones and Józef H. Przytycki. Lissajous knots and billiard knots, Knot theory (Warsaw, 1995), Banach Center Publ., 42:145-163. Polish Acad. Sci. Inst. Math., Warsaw, 1998.

[35] Louis H. Kauffman. Formal knot theory, Mathematical Notes, 30. Princeton University Press, Princeton, NJ, 1983.

[36] _ . A Tutte polynomial for signed graphs. Discrete Appl. Math. 25(1-2):105-127, 1989.

[37] Richard Kenyon. Lectures on dimers. Statistical mechanics, IAS/Park City Math. Ser., 16:191-230. Amer. Math. Soc., Providence, RI, 2009.

[38] Richard W. Kenyon, James G. Propp, and David B. Wilson. Trees and matchings. Electron. J. Combin., 7:34pp., (2000).

[39] P.-V. Koseleff and D. Pecker. On polynomial torus knots. J. Knot Theory Ramifications, 17(12):1525-1537, 2008.

[40] Chebyshev diagrams for two-bridge knots. Geom. Dedicata, 150:405-425, 2011.

[41] Chebyshev knots. J. Knot Theory Ramifications, 20(4):575-593, 2011.

[42] Harmonic knots. arXiv: 1203.4376v1, 2012.

[43] Dmitry N. Kozlov. Discrete Morse theory for free chain complexes. C. R. Math. Acad. Sci. Paris, 340(12):867-872, 2005.

[44] Olga Kravchenko and Michael Polyak. Knots, dimers, and cluster algebras. Personal communication, 2014.

[45] Christoph Lamm. There are infinitely many Lissajous knots. Manuscripta Math., 93(1):29-37, 1997.

[46] Z Z Zylinder-Knoten und symmetrische Vereinigungen. Bonner Mathematische Schriften [Bonn Mathematical Publications], 321. Dissertation, Rheinische FriedrichWilhelms-Universität Bonn. Universität Bonn Mathematisches Institut, Bonn, 1999.

[47] W. B. Raymond Lickorish. An introduction to knot theory, Graduate Texts in Mathematics, 175. Springer-Verlag, New York, 1997. 
[48] L. Lovász. Combinatorial problems and exercises. North-Holland Publishing Co., Amsterdam, 1979.

[49] L. Lovász and M. D. Plummer. Matching theory, North-Holland Mathematics Studies, 121. North-Holland Publishing Co., Amsterdam, 1986.

[50] Francesca Mori and Mario Salvetti. (Discrete) Morse theory on configuration spaces. Math. Res. Lett., 18(1):39-57, 2011.

[51] Peter Ozsváth and Zoltán Szabó. Heegaard Floer homology and alternating knots. Geom. Topol., 7:225-254, 2003.

[52] M. D. Plummer. On n-extendable graphs. Discrete Math., 31(2):201-210, 1980.

[53] Lawrence Roberts. Totally twisted Khovanov homology. arXiv:1109.0508v1, 2011.

[54] Morwen B. Thistlethwaite. A spanning tree expansion of the Jones polynomial. Topology, 26(3):297-309, 1987.

[55] S. Wehrli. A spanning tree model for Khovanov homology. J. Knot Theory Ramifications, 17(12):1561-1574, 2008.

[56] Volkmar Welker. Discrete Morse theory and free resolutions, Algebraic combinatorics, 81-172. Universitext, Springer, Berlin, 2007.

[57] Heping Zhang and Fuji Zhang. Plane elementary bipartite graphs. Discrete Appl. Math. 105(1-3):291-311, 2000. 